Prepared for the U.S. Department of Energy

under Contract DE AC05 76RL01830

\title{
Analysis of ISO NE Balancing Requirements: Uncertainty-based Secure Ranges for ISO New England Dynamic Interchange Adjustments
}

PV Etingov
D Wu
Y Sun
X Luo
S George
E Litvinov
E Sanchez

YV Makarov

Z Hou

S Maslennikov

T Zheng

T Knowland

S Weaver

January 2013

Pacific Northwest

NATIONAL LABORATORY

Proudly Operated by Battelle Since 1965 


\title{
DISCLAIMER
}

This report was prepared as an account of work sponsored by an agency of the United States Government. Neither the United States Government nor any agency thereof, nor Battelle Memorial Institute, nor any of their employees, makes any warranty, express or implied, or assumes any legal liability or responsibility for the accuracy, completeness, or usefulness of any information, apparatus, product, or process disclosed, or represents that its use would not infringe privately owned rights. Reference herein to any specific commercial product, process, or service by trade name, trademark, manufacturer, or otherwise does not necessarily constitute or imply its endorsement, recommendation, or favoring by the United States Government or any agency thereof, or Battelle Memorial Institute. The views and opinions of authors expressed herein do not necessarily state or reflect those of the United States Government or any agency thereof.

\author{
PACIFIC NORTHWEST NATIONAL LABORATORY \\ operated by \\ BATTELLE \\ for the \\ UNITED STATES DEPARTMENT OF ENERGY \\ under Contract DE-AC05-76RL01830 \\ Printed in the United States of America \\ Available to DOE and DOE contractors from the \\ Omice of Scientific and Technical Information. \\ P.O. Box 62, Oak Ridee, TN 37831-0062: \\ ph: (865) 576-8401 \\ fax: $(865) 576-5728$ \\ email: reports $a$ adonisosti.gov \\ Available to the public from the National Technical Information Service \\ 5301 Shawnee Rd., Alexandria, VA 22312 \\ ph: (800) 553.NTIS (6847) \\ email: ordersantis,gov 〈http:/www.ntis.gov/abeat/form.aspx> \\ Online ordering: http:/www.ntix.gov
}

कैै This document was printed on recycled paper.

(82010) 


\section{Analysis of ISO NE Balancing Requirements: Uncertainty-based Secure Ranges for ISO New England Dynamic Interchange Adjustments}

$\begin{array}{ll}\text { PV Etingov } & \text { YV Makarov - Project Manager } \\ \text { D Wu } & \text { Z Hou } \\ \text { Y Sun } & \text { S Maslennikov } \\ \text { X Luo } & \text { T Zheng } \\ \text { S George } & \text { T Knowland } \\ \text { E Litvinov } & \text { S Weaver } \\ \text { E Sanchez } & \end{array}$

January 2013

Prepared for

U.S. Department of Energy

under Contract DE-AC05-76RL01830

Pacific Northwest National Laboratory

Richland, Washington 99352 



\begin{abstract}
Dynamic adjustments of the interchange between Independent System Operator - New England (ISO-NE) and neighboring areas will enable these transmission system operators to trade balancing services and consequently reduce operational costs. This can be achieved through efficient utilization of available internal and external flexible generation resources and available ramping capability.

This report is prepared to detail the work conducted for ISO-NE by Pacific Northwest National Laboratory (PNNL) under contract 1953-0-SOS (PNNL 62527). The document describes detailed uncertainty quantification (UQ) methodology developed by PNNL to estimate secure ranges of potential dynamic intra-hour interchange adjustments in the ISO-NE system and provides description of the dynamic interchange adjustment (DINA) tool developed under the same contract. The overall system ramping up and down capability, spinning reserve requirements, interchange schedules, load variations and uncertainties from various sources that are relevant to the ISO-NE system are incorporated into the methodology and the tool. The DINA tool has been tested by PNNL and ISO-NE staff engineers using ISO-NE data.
\end{abstract}

ISO-NE will continue testing DINA to evaluate its performance and applicability to ISO-NE operational needs. 



\section{Summary}

Currently, the ISO-NE net interchange is scheduled on the hourly basis for 1-hour intervals. In the near future, ISO-NE plans to implement intra-hour 4-hour-ahead dispatch. In particular, ISO-NE plans to implement 15-minute dispatch intervals for intertie flow with New York Independent System Operator (NY-ISO) in response to the Federal Energy Regulatory Commission (FERC) Order No. 764, released on June 22, 2012. This order requires grid operators to allow 15-minute scheduling of transmission service as an alternative to the existing 1-hour scheduling process [FERC 2012]. FERC requires balancing authorities (BAs) to comply FERC Order requirements within 12 months of the Order's effective date. The change strongly encourages balancing authorities to more accurately and more frequently schedule energy balance and reserve requirements. The more granular scheduling could lead to the development of new balancing products and forms of cooperation between BAs. To successfully realize this approach, the ISO-NE dispatchers need to know the secure range for potential interchange adjustments, for both incremental and decremental changes, which would result in a minimum risk of potential imbalances within this system. It is therefore extremely important to know the secure range of potential intra hour interchange adjustments for both incremental and decremental changes such that the changes would result in a minimum risk of potential imbalances in the system. This is the issue addressed by this project.

The secure interchange adjustment range can be predicted ahead of time. It depends on the overall upward and downward ramping capability of the system, internal balancing requirements caused by changing net load and interchange, spinning reserve requirements, and available transfer capability on the interties. A significant part of this information is unknown ahead of time under the current ISO-NE operational conditions, so the secure interchange adjustment range cannot be accurately quantified using deterministic methods. There are several uncertainties to take into account when estimating the potential interchange adjustment by ISO-NE:

- Generation upward and downward ramping capability (it depends on the future unit commitment and dispatch, which are unknown at the moment of prediction),

- $\quad$ System load forecast errors,

- Uninstructed deviations of generators from their set points, and

- Future system spinning reserve requirements.

The project objective is to develop a dynamic interchange adjustment (DINA) tool, which provides an online estimation of the secure ISO-NE net interchange variation ranges within the next dispatch interval (for 45 and 65 minutes ahead of the current time), with user-specified confidence levels. The DINA tool considers all uncertainties influencing this estimation.

The methodology includes the following main elements.

(1) A methodology to determine the maximum system ramping capability for the next operating hour, developed by ISO-NE engineers. The algorithm reflects the most important characteristics influencing the maximum possible upward and downward interchange 
increments within 45 and 65 minutes ahead of the current time (10 and 30 minutes after the beginning of the ramp connecting the preceding hour with the analyzed operating hour):

- Ramping characteristics of online dispatchable generators;

- Their dispatch points and available upward and downward capacity;

- Available fast start units currently offline;

- $\quad$ Spinning reserve requirements (for the upward ramping characteristics);

- Load increase or decrease, and

- Interchange variation.

(2) A procedure to quantify the future uncertainties and to determine the secure range of possible ISO-NE intra-hour interchange adjustments. The proposed uncertainty quantification procedure is based on a comparison of the predicted and the actual ramping capability characteristics of the ISO-NE system and collecting statistics on the differences over an observation period in the past, $\mathrm{T}$.

a. The predicted upward and downward ramping capability of ISO-NE system is calculated based on the information available at the moment of prediction. The predicted a priori range is affected by uncertainties of the generation dispatch, load forecast, interchange variations, and reserve requirements.

b. The actual range is calculated based on information available after the analyzed hour ends. This a posteriori range is known exactly for the past interval.

c. Then we compare the upward and downward actual versus predicted assessments for 45 and 65 minutes ahead, and calculate the differences in the upward and downward requirements for these points.

d. We accumulate sets of these differences over the past interval T, e.g., over 1 or 3 months. These sets are calculated separately for each operating hour of the day, and separately for the business days and weekends.

e. The next step is to build probability distributions for each of the sets over period T.

f. Based on a user-specified confidence level, the uncertainty of interchange range (in MW) can be calculated. For example, one can evaluate the upper and lower uncertainty bounds with $95 \%$ confidence by cutting $5 \%$ of the extreme points on one of the ends of each probability distribution.

(3) Assessment of the ramping up and ramping down capability of the system. In real time, when we predict the secure range for interchange adjustments, we first assess the ramping up and ramping down capability of the system based on limited information available at the current moment. Then we simply reduce the predicted adjustment range by uncertainty margin 
evaluated in step 2. The margin is different for different hours of an operating day, as well as for business and weekend dates.

(4) A process for automatic self-validation of UQ results enables system operators to evaluate the performance of the DINA tool. The self-validation procedure includes comparing the actual (observed) system capability with predicted uncertainty ranges, and calculating the percentage of actual points within the predicted range.

(5) A software tool for interchange requirements uncertainty quantification has been developed. It is based on the methodology outlined in this report. The DINA tool consists of three main modules (tiers): the database, the generation capability and uncertainty evaluation algorithm, and the graphical user interface. Initial version of the DINA tool was installed at the ISO-NE for testing in November 2012.

(6) Trial runs have been conducted to test the uncertainty assessment approach and to evaluate and demonstrate capabilities of the DINA tool. ISO-NE 2011 data were used in the simulation and the tool development. The dataset provided by ISO-NE included: market information on available generation characteristics and dispatch, actual load, load forecast, actual interchange, interchange schedule, and actual generation. Simulation results received preliminary evaluation by ISO-NE engineers. ISO-NE will continue testing the DINA tool using more data. Assuming the extended testing is successful, ISO-NE can decide on the actual use of the tool and its integration with ISO-NE systems.

In this project, PNNL has been working with a support team of ISO-NE experts.

Dynamic adjustments of the interchange between ISO-NE and neighboring areas will enable these transmission system operators (TSOs) to trade balancing services and consequently reduce operational costs. This can be achieved through efficient utilization of available internal and external flexible generation resources and available ramping capability. 



\section{Acknowledgments}

The authors would like to thank ISO New England (ISO-NE) who sponsored and supported this project and provided data, advice, managerial, technical and organizational assistance:

- ISO-NE project managers:

o Mr. Daniel Charko, Project Manager

o Mr. Krishnamohan Kumsi, Project Manager, Project Management Office

The authors would also like to thank the following PNNL staff for their support and guidance:

- Dr. Krishnappa Subbarao, Senior Engineer

- Dr. Landis D. Kannberg, Lead Manager - Energy Storage,

- Mr. Carl H. Imhoff, Manager, Electricity Infrastructure Market Sector,

- Mr. Dale A. King, Manager, Energy Science and Technology Product Line,

- Dr. Mark P. Morgan, Manager, Advanced Power \& Energy Systems Group,

- Dr. Ronald B. Melton, Team Lead, Electrical Power Systems Integration Group,

- Ms. Sheena L. Kanyid, Contracting Officer,

- $\quad$ Ms. Susan J. Arey, Project Specialist, and

- $\quad$ Ms. Nikki R. Stringer, Administrative Assistant. 



\section{Acronyms and Abbreviations}

AEMN

AEMX

AGC

BA

Battelle

Case ID

CAISO

$\mathrm{CDF}$

Claim 10

Claim 30

CRR

DDP

DINA

DLL

DOE

EcoMax

EcoMin

FERC

GUI

ID

ISO-NE

LEG

LEGFLAG

MRR

MW

NERC

NY ISO

PDF

Pmax

Pmin

PNNL
Economic minimum of generation

Economic maximum of generation

Automatic generation control

Balancing authority

Battelle Memorial Institute

Automatically generated stamps including the date and time of the corresponding data points

California Independent System Operator Corp.

Cumulative distribution function

The generation output level, expressed in megawatts, that a generator can reach from an off-line state in 10 minutes after receiving a dispatch instruction [ISONE Glossary].

The generation output level, expressed in megawatts, that a generator can reach from an off-line state in 30 minutes after receiving a dispatch instruction [ISONE Glossary].

Claimed Ramp Rate

Desired dispatch point for generators

Dynamic interchange adjustment

Dynamic-link library

U.S. Department of Energy

Economic maximum of generation

Economic minimum of generation

Federal Energy Regulatory Commission

Graphical user interface

Identification

Independent System Operator - New England, Inc.

Limited energy generator

A flag for limited energy generators (1 - LEG; 0 - Not LEG)

Minimum ramp rate, $\mathrm{MW} /$ minute

Megawatt

North American Electric Reliability Corporation

New York Independent System Operator

Probability distribution function

Maximum output of a generation unit

Minimum output of a generation unit

Pacific Northwest National Laboratory 
SCED

STD

TSO

UCM

UQ

W
Security-constrained economic dispatch

Standard deviation

Transmission system operator

Unit control mode

Uncertainty quantification

Watt 


\section{Contents}

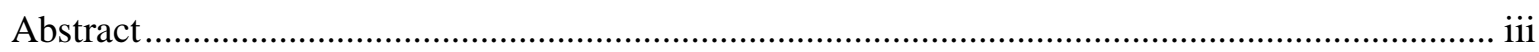

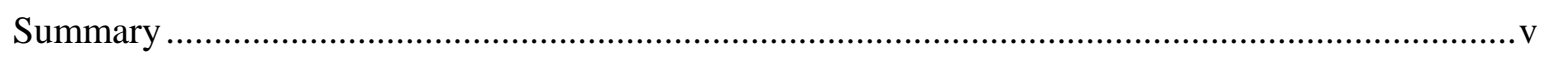

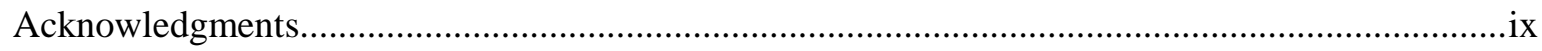

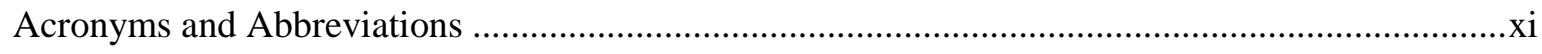

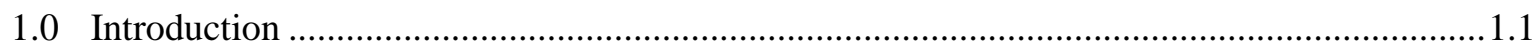

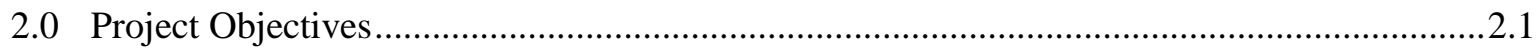

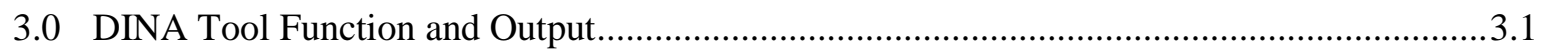

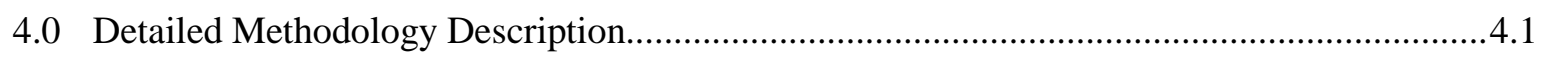

4.1 Calculation of Upward and Downward Maximum Ramping Characteristics..................4.1

4.1.1 Generation Up and Down Maximum Ramping Characteristics..........................4.1

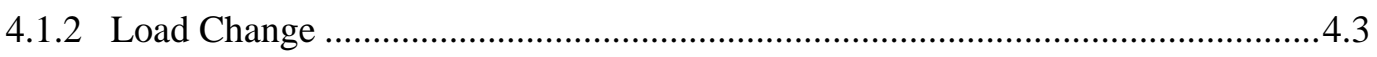

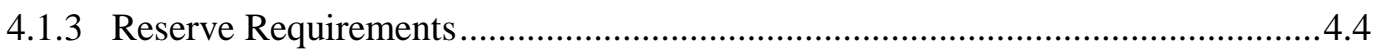

4.2 Quantification of Resulting Uncertainty Margins ......................................................4.4

4.3 “Look-ahead” Estimation in Real Time .......................................................................9

4.4 Validation Procedure for Results ................................................................................. 4.11

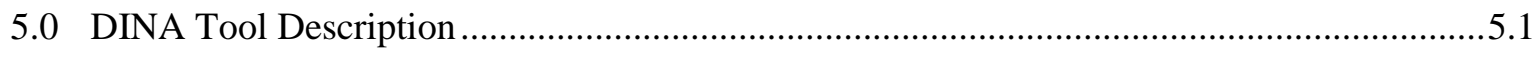

5.1 Software Requirements and Structure ................................................................. 5.1

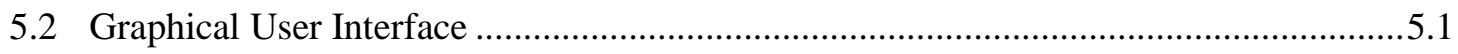

5.2.1 Main Display ........................................................................................... 5.2

5.2.2 Generation Ramping Capability Screen ......................................................... 5.3

5.2.3 Interchange Limits Prediction Errors Statistics Screen ........................................5.5

5.2.4 Load Forecast, Interchange and Generation Errors Statistics Screen....................5.6

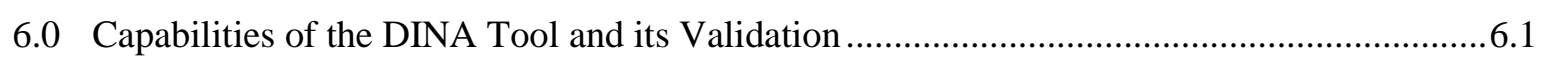

6.1 Generation Ramping Capability ................................................................................. 6.1

6.2 Secure Interchange Adjustment Range ….................................................................. 6.5

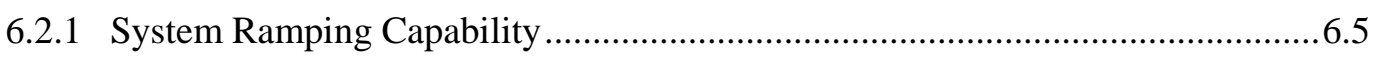

6.2.2 Prediction Errors Analysis .............................................................................6.6

6.2.3 Probabilistic System Capability …................................................................. 6.7

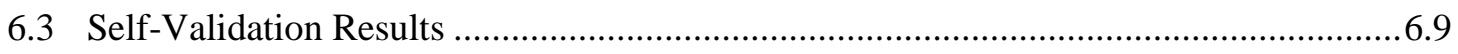

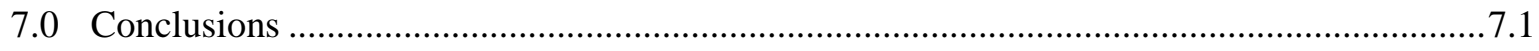

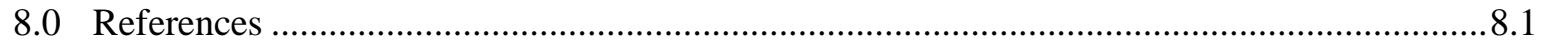

Appendix A . ISO-NE Ramping Capability Calculation Methodology .........................................1 


\section{Figures}

Figure 3-1. Secure range for interchange variations 3.1

Figure 4-1. Ramping up capability of a unit with multi-segment ramping characteristic.

Figure 4-2. Ramping capability of an example online unit.

Figure 4-3. Predicted and actual upward and downward ramping capability..........

Figure 4-4. Predicted and actual upward and downward ramping capability

Figure 4-5. Building CDF for each variable.....

Figure 4-6. Calculation of: (a) Upward uncertainty range prediction, $\Delta^{\mathrm{UP}} b$, and (b) Downward uncertainty range prediction, $\Delta^{\mathrm{DN}} b$

Figure 4-7. Estimation of the secure interchange adjustment range

Figure 4-8. Example of actual interchange adjustment range estimation

Figure 4-9. Histograms of prediction errors for upward system capability at hour ending by 1 for (a) $t_{0}+45$; (b) $t_{0}+65$

Figure 4-10. Histograms of prediction errors for downward system capability at hour ending by 1

for (a) $t_{0}+45$; (b) $t_{0}+65$

Figure 4-11. Self-validation results

Figure 5-1. The software structure

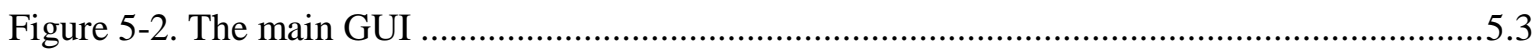

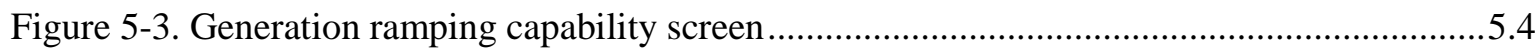

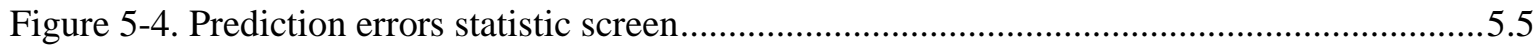

Figure 5-5. Load forecast, interchange and generation schedule errors statistics...........................5.7

Figure 6-1. Ramping characteristic of online dispatchable unit. ...............................................6.3

Figure 6-2. Ramping characteristic of an offline fast-start unit .....................................................6.3

Figure 6-3. Ramping characteristic of the unit with ramp segments ...........................................6.4

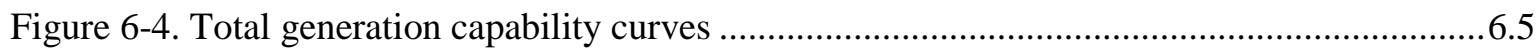

Figure 6-5. System capability: (a) Online and Offline units; (b) Online units only .......................6.6

Figure 6-6. System capability prediction errors analysis .........................................................6.7

Figure 6-7. Secure Interchange adjustment range: (a) Online and Offline units; (b) Online units only

Figure 6-8. Self-validation results 


\section{Tables}

Table 4-1. Statistical characteristics of prediction errors for the upward and downward

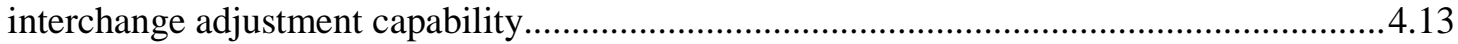

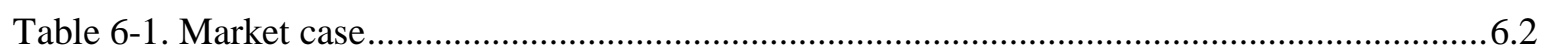

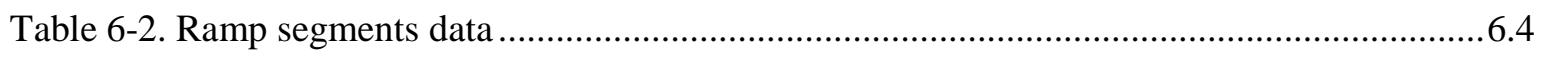

Table 6-3. Prediction errors statistics............................................................................................6.6 



\subsection{Introduction}

On June 22, 2012, the Federal Energy Regulatory Commission (FERC) released Order No. 764 requiring grid operators to allow 15-minute scheduling of transmission service [FERC 2012]. FERC requires compliance within 12 months of the Order's effective date. The intra-hour scheduling process motivates balancing authorities (BAs) to more accurately plan energy balance and reserve requirements. The more granular scheduling could lead to the development of new balancing products and forms of BA cooperation.

Currently, ISO-NE dispatch is based on 5-minute security-constrained economic dispatch (SCED) with generation dispatch signals created by market applications for up to 15 minutes ahead of time. Net interchange flow is scheduled hourly for 1 hour ahead of time. Ramps between the hourly schedules are linear and have a 10-minute duration. The scheduling process starts about 40 minutes before the end of the hour and takes about 10 to 20 minutes. In the near future, ISO-NE will establish multi-interval intrahour dispatch for up to 4 hours ahead of time and 15-minute dispatch intervals for intertie flow with New York Independent System Operator (NY ISO).

Dynamic intra-hour adjustment of interchange between systems enables system operators with the opportunity to trade the balancing energy and consequently reduce their balancing reserve requirements as well as operational costs by more efficient utilization of available internal and external flexible generation resources and available transfer capability. At the same time, the interchange adjustments, which are planned in near-real-time, must stay within certain security limits to avoid potential imbalances. The possible secure interchange adjustment range can be predicted ahead of time and is dependent on the upward and downward ramping capability of the system, internal balancing requirements caused by changing net load and interchange, spinning reserve requirements, and available transfer capability on the interties, which in its own turn is limited by transmission thermal constraints and potentially stability constraints. A significant part of this information is unknown ahead of time and therefore uncertain, so it cannot be quantified using existing deterministic methods and tools. Among these uncertainties that need to be taken into account when estimating the potential interchange adjustment at ISO-NE are the following factors: (1) generation upward and downward ramping capability because it depends on the unit commitment and dispatch, which are unknown at the moment of prediction, (2) system load variations caused by forecast errors, (3) uninstructed deviations of generation units, and (4) future system spinning reserve requirements.

In this project, PNNL worked with the ISO-NE support team to develop a DYNA methodology and software tool capable of estimating the secure interchange adjustment range of the tie line schedule based on statistical uncertainty quantification (UQ) methods. This report presents the detailed methodology, its implementation in the developed DINA tool, as well as test results based on ISO-NE 2011 data. 



\subsection{Project Objectives}

The PNNL dynamic interchange adjustment (DINA) tool is designed to provide an online estimation of the secure range for possible ISO-NE intra-hour net interchange adjustments within the next dispatch interval, for 45 and 65 minutes ahead of time, with user-specified confidence levels. The DINA tool considers all contributing factors, such as expected changes to system load and interchange, spinning reserve requirements, and system ramping capability, as well as relevant uncertainties significantly impacting this estimation, including load forecast errors, random interchange variations, uninstructed deviations on generators, uncertainties in generation dispatch and reserve requirements. The DINA tool uses ISO-NE market and other data in a form as agreed upon with NE-ISO. 



\subsection{DINA Tool Function and Output}

ISO-NE dispatch is based on 5-minute security-constrained economic dispatch (SCED) with generation dispatch signals created by market applications for up to 15 minutes ahead of time. Net interchange flow is scheduled hourly for 1 hour ahead of time. Ramps between the hourly schedules are linear and have a 10-minute duration. The scheduling process starts about 40 minutes before the end of the hour and takes about 10 to 20 minutes $^{1}$.

The DINA tool predicts the secure range of interchange dynamic adjustments from its hourly schedule (orange dots and lines in Figure 3-1) for 45 and 65 minutes ahead of time with user-specified level of confidence, in upward $\left(\Delta_{45}{ }^{\mathrm{UP}}, \Delta_{65}{ }^{\mathrm{UP}}\right)$ and downward $\left(\Delta_{45}{ }^{\mathrm{DN}}, \Delta_{65}{ }^{\mathrm{DN}}\right)$ directions. All time and confidence level settings in the tool are adjustable, so that it will be easy to accommodate any future changes in the timeline and intervals.

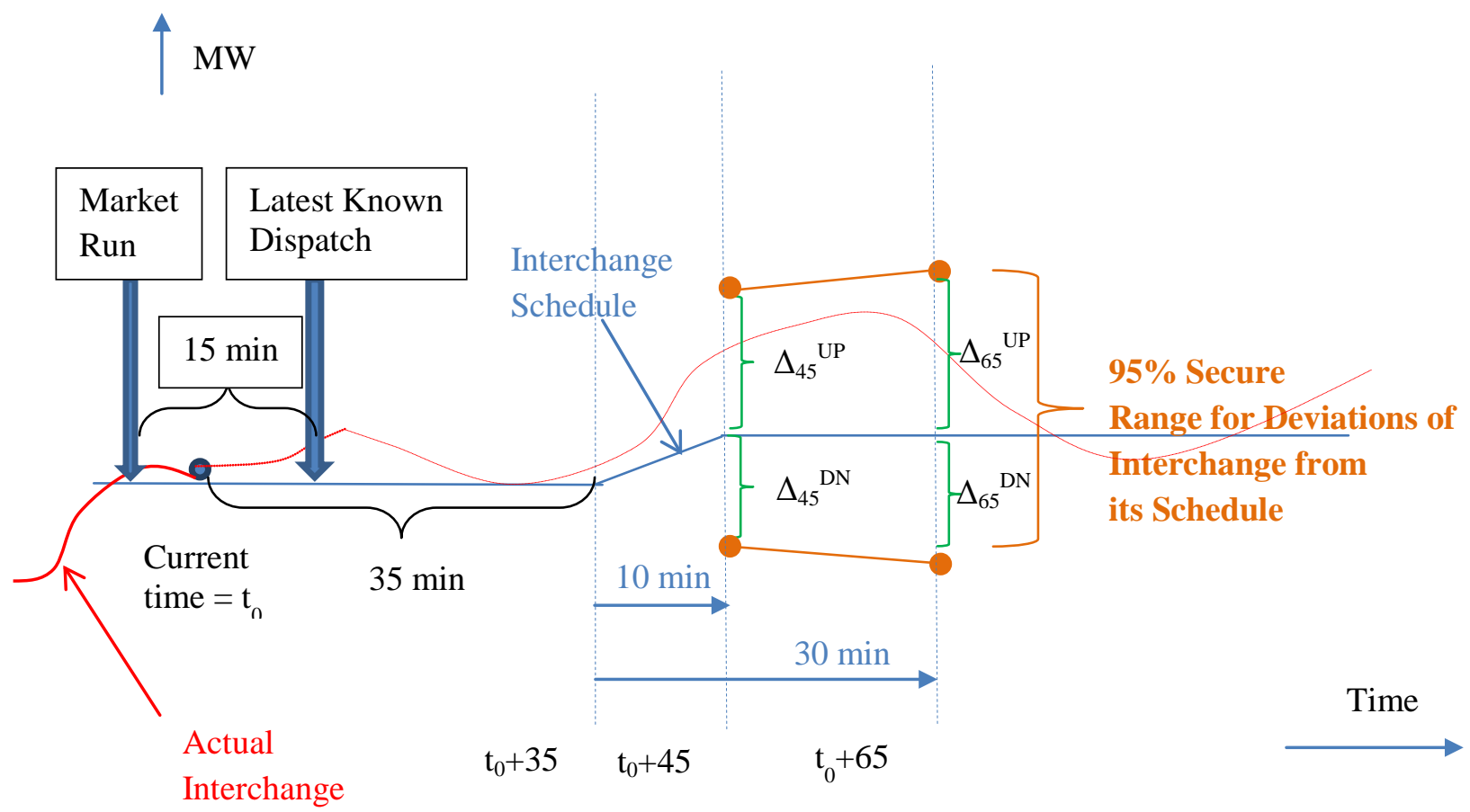

Figure 3-1. Secure range for interchange variations

\footnotetext{
${ }^{1}$ Expected future change, which is not in the scope of this project: ISO-NE is working to establish multi-interval dispatch for up to 4 hours look-ahead. Another development target is to create 15-minute dispatch intervals for interchange flow with NY ISO.
} 



\subsection{Detailed Methodology Description}

This section describes the detailed methodology developed and used in this project for calculating the next secure intra-hour interchange adjustments for the next operating hour.

\subsection{Calculation of Upward and Downward Maximum Ramping Characteristics}

At a given time, $t_{0}$, the most important characteristics influencing the maximum upward and downward points at $t_{0}+45$ and $t_{0}+65$ are:

(1) Ramping characteristics of online dispatchable generators, MW/min;

(2) Their dispatch points and available upward and downward capacity, MW;

(3) Available fast starting units that are currently offline;

(4) Operating reserve requirements (they are important for the upward ramping characteristics), MW;

(5) Load increase or decrease at $t_{0}+45$ and $t_{0}+65$ starting from the current time $t_{0}$, MW, and

(6) Interchange variation at $t_{0}+45$ and $t_{0}+65$ starting from the current point $t_{0}$, MW.

\subsubsection{Generation Up and Down Maximum Ramping Characteristics}

The calculation of upward and downward ramping characteristics is conducted based on the methodology developed by ISO New England - see its full description in Appendix A. In a high-level condensed form, the methodology for generation up and down maximum characteristics can be outlined as follows.

Both dispatchable online units and available offline fast-start units contribute to the system ramping capability. For each online unit, its ramping capability is determined by its specific ramping characteristics and dispatch status, such as maximum and minimum generation, maximum ramping rate, and current dispatch point. Let $P_{\text {gen }}^{i}(t)$ denotes generation dispatch of unit $i$ at time $t$. The upper and lower bounds of power output from this unit at time $t_{0}+\Delta t$, where $\Delta t=45$ or 65 minutes $^{1}$, can be calculated as:

$$
\begin{aligned}
& P_{\text {gen_up }}^{i}\left(t_{0}+\Delta t\right)=\min \left\{P_{\text {gen }}^{i}\left(t_{0}+35\right)+R_{i} \times(\Delta t-35), P_{\max }^{i}\right\} \\
& P_{\text {gen_dn }}^{i}\left(t_{0}+\Delta t\right)=\max \left\{P_{\text {gen }}^{i}\left(t_{0}+35\right)-R_{i} \times(\Delta t-35), P_{\min }^{i}\right\}
\end{aligned}
$$

\footnotetext{
${ }^{1}$ At this point, it should be reminded that $t_{0}$ is the point where a decision is made regarding available secure range for possible ISO-NE interchange adjustments ("current point"); $t_{0}+35$ is the "starting point" where the ramp between the current and the next operating hour begins; $t_{0}+45$ is the point where the hourly ramp ends (this is the first point where the interchange schedule can be dynamically adjusted, and $t_{0}+65$ is the second intra-hour adjustment point specified by ISO-NE.
} 
where $R_{i}$ is the unit's ramp rate, and $P_{\max }^{i}$ and $P_{\min }^{i}$ are its maximum and minimum generation, respectively.

Some unit ramping characteristics can have segments with different ramp rates ${ }^{1}$. For example, Figure 4-1 illustrates the upper bound of a unit's multi-segment power output.

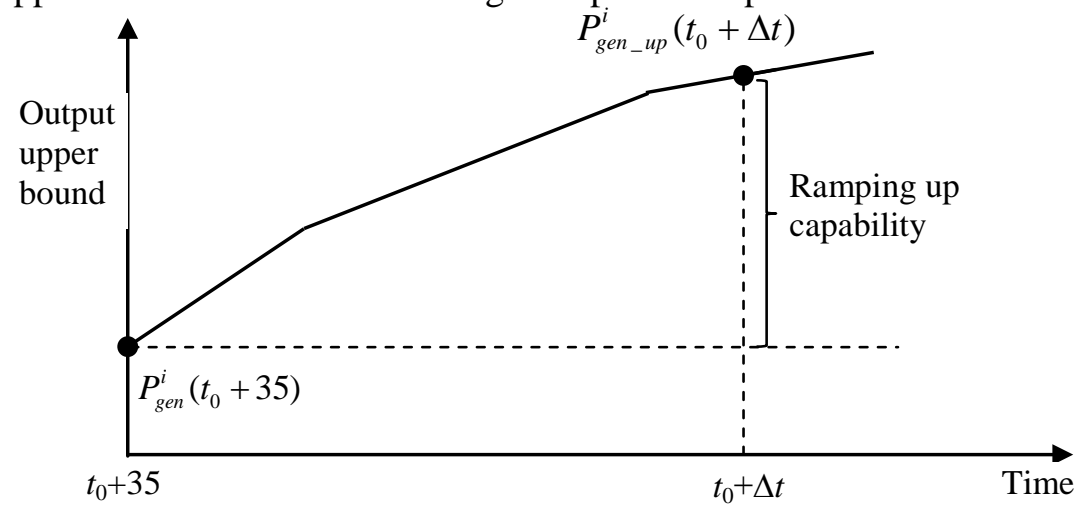

Figure 4-1. Ramping up capability of a unit with multi-segment ramping characteristic

For each available offline fast start unit, there is no ramp-down capability; therefore, only ramp-up capability needs to be calculated. Generally, MW claims of the startup ramping up capability within certain time duration are provided by offline units. (E.g., "claim 10"2 and "claim 30"3 represent the MW output that can be made provided by a unit within 10 and 30 minutes after startup instruction is issued, that is 45 and 65 minutes from the point where ISO-NE makes this decision). For the fast-startup units, this MW claim replaces the term $P_{\text {gen }}^{i}\left(t_{0}+35\right)+R_{i} \times(\Delta t-35)$ in (1), used to calculate the upper bound of the power output of online units.

There are also units with limited energy resource, thus adding another potentially more limiting constraint to the upper bounds of outputs from these units - see Appendix A for details.

An example of real unit ramping capability characteristic (a screen shot of the tool) is shown in Figure 4-2.

\footnotetext{
${ }^{1}$ Note that the mathematical description of multi-segment characteristic is more complicated than the one given in equations (1) and (2). We are not providing this description in this report.

${ }^{2}$ Claim 10 - The generation output level, expressed in megawatts, that a generator can reach from an off-line state within 10 minutes after receiving a dispatch instruction from ISO. This value is required as part of the generator's offer data and is used by ISO New England to evaluate the generator's contribution towards meeting operating- and replacement-reserve requirements [ISO-NE Glossary].

${ }^{3}$ Claim 30 - The generation output level, expressed in megawatts, that a generator can reach from an off-line state in 30 minutes after receiving a dispatch instruction from ISO. This value is required as part of the generator's offer data and is used by ISO New England to evaluate the generator's contribution towards meeting the New England Control Area's operating and replacement reserve requirements [ISO-NE Glossary].
} 


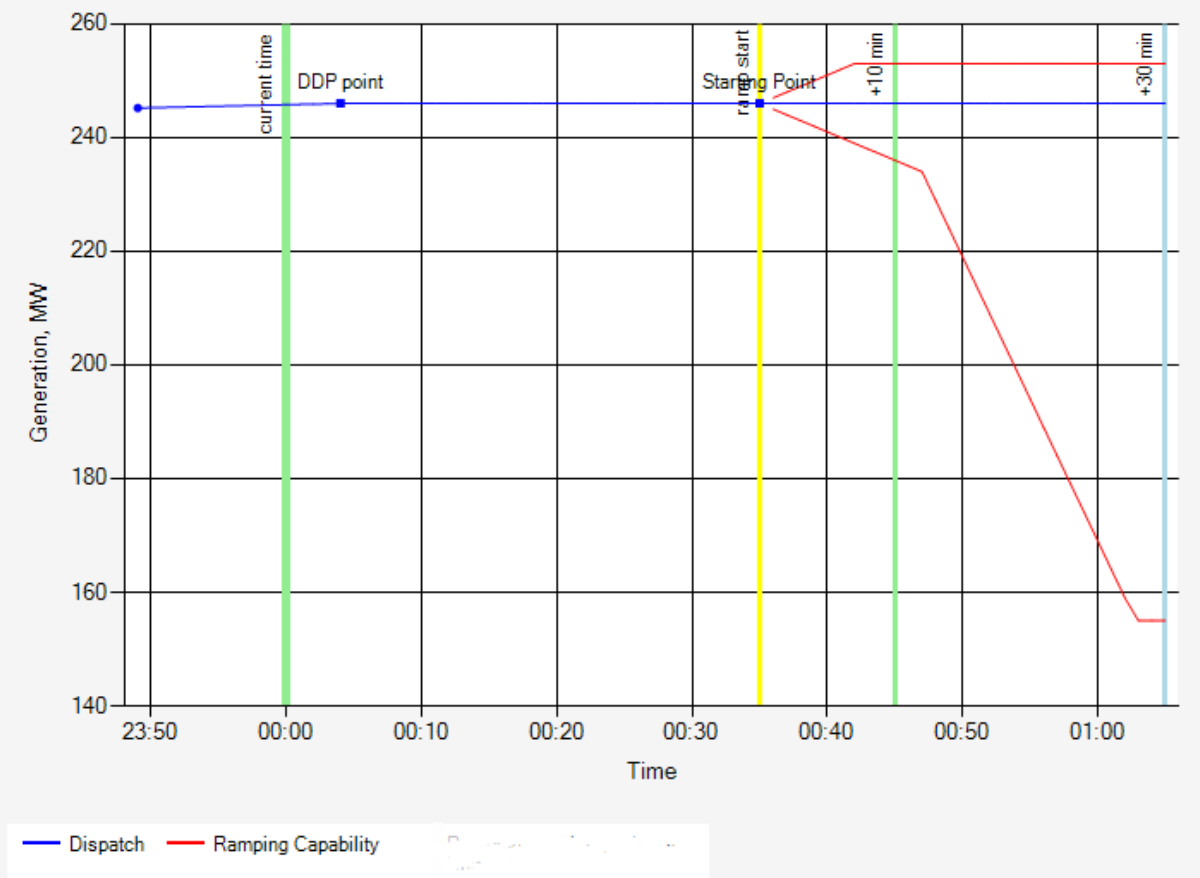

Figure 4-2. Ramping capability of an example online unit

In Figure 4-2, "DDP” is the desired dispatch point; "starting point” where the ramp between the current and the next operating hour begins, $t_{0}+35$; " 10 min" and "20 min" mark the moments where the interchange can be adjusted, $t_{0}+45$ and $t_{0}+65$, correspondingly).

The overall system upward and downward generation capability characteristics are obtained by summing up the capabilities for all the units available for redispatch or startup.

Unfortunately, the unit dispatch at $t_{0}+35$ is not known at $t_{0}$, and the best information that corresponds to the latest market run conducted before $t_{0}$ and predicting the unit status for only 15 minutes ahead. If we use this information for our analysis at $t_{0}+35$, we introduce additional uncertainty. This uncertainty can be quantified by comparing the predicted ramping characteristic at $t_{0}$ with the actual characteristic assessed at $t_{0}+65$, that is after the fact, for some past interval $\mathrm{T}$.

The functions calculating the maximum ramping capability for the moments $t_{0}+45$ and $t_{0}+65$ can be denoted as RampUp $\left(t_{0}+45\right)$, RampDn $\left(t_{0}+45\right)$, RampUp $\left(t_{0}+65\right)$, and $\operatorname{RampDn}\left(t_{0}+65\right)$. These functions are ran twice to produce the predicted and actually available interchange adjustment ranges used in our methodology, but with different combinations of input parameters, as described below.

\subsubsection{Load Change}

The change of load also affects the tie line ramping capability calculation. For instance, a load increase from $t_{0}$ to $t_{0}+45$ and further to $t_{0}+65$ reduces system upward ramping capability, and at the same time, it increases system downward ramping capability. In the proposed method, we use the forecasted load and actual variations in the system ramping capability calculation to evaluate the uncertainties in forecasted load values versus actual values. 


\subsubsection{Reserve Requirements}

Dynamic interchange adjustments should not compromise system reliability performance. Based on operational reliability requirements, some of the spinning generation capacity must be reserved as operating reserve and cannot be used for interchange adjustments. Hence, the reserve requirement needs to be subtracted from system's generation upward capability while calculating the secure range of interchange variations. At $t_{0}+35$, ISO-NE dynamically calculates the reserve requirements that need to be activated in 10 and 30 minutes from $t_{0}+35$ (at $t_{0}+45$ and $t_{0}+65$ ), but they are not known at the current point $t_{0}$. This circumstance creates additional uncertainty addressed in the proposed methodology by quantifying historically observed differences between the reserve requirements assessed in the market runs right before $t_{0}$ and the actual reserve requirements at $t_{0}+45$ and $t_{0}+65$.

\subsection{Quantification of Resulting Uncertainty Margins}

The proposed uncertainty quantification procedure is based on a comparison of the predicted (assessed at $t_{0}$ ) and the actual (assessed after the fact at $t_{0}+65$ ) ramping capability characteristics of the ISO-NE system for the moments $t_{0}+45$ and $t_{0}+65$.

The predicted up and down ramping capabilities (green dots and lines in Figure 4-3) are calculated based on the information available at $t_{0}$. The predicted a priori range is affected by uncertainties of generation dispatch, load forecast, interchange variations, and reserve requirements.

The actual up and down ranges (red dots and lines in Figure 4-3) are calculated based on a posteriori information available at $t_{0}+65$. This range is known exactly for the past interval $\mathrm{T}$. 


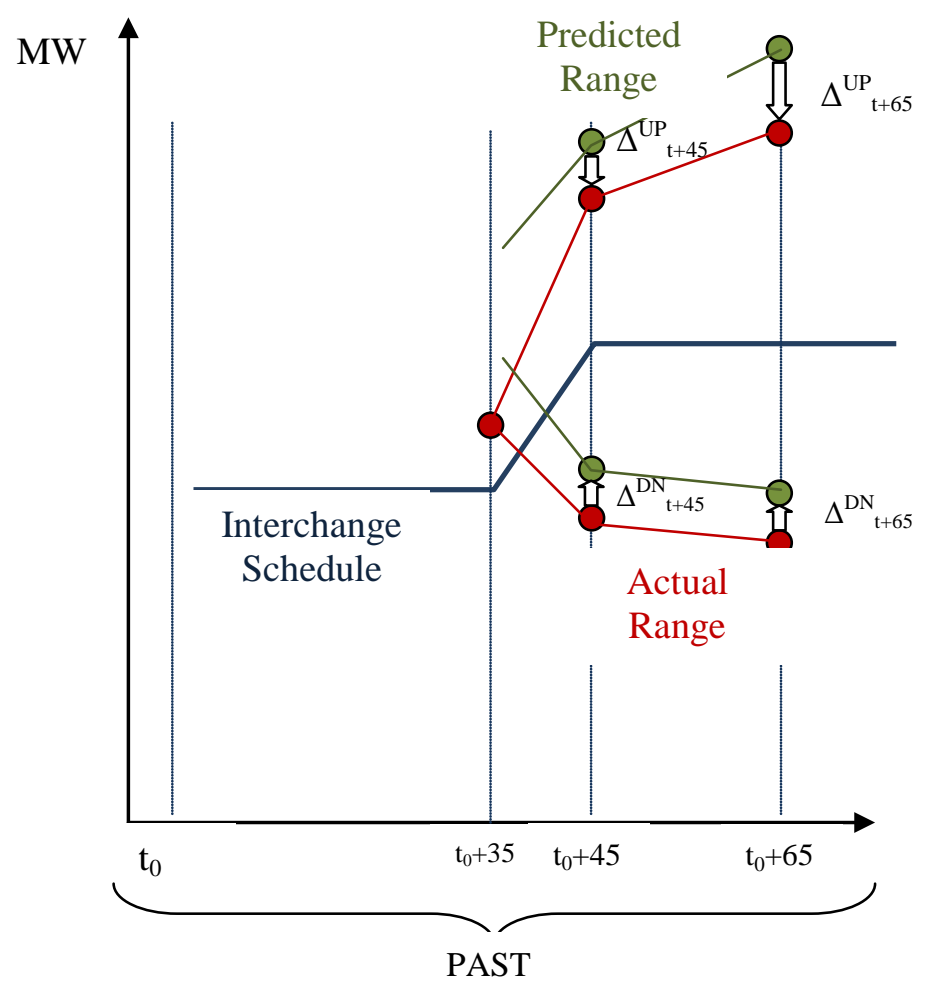

Figure 4-3. Predicted and actual upward and downward ramping capability

The past interval $\mathrm{T}$ is defined as

$$
t_{0}-\mathrm{T}-65<t<t_{0}-65 \text {, min, }
$$

where $t_{0}$ is the current time, and $\mathrm{T}$ is a configurable interval.

For the "predicted" ramping capability, calculated for intervals within the past period T, we use the following information -see Figure 4-4:

(a) The forecasted load change $\Delta \mathrm{L}_{\mathrm{f}}, \Delta \mathrm{L}_{\mathrm{f}}\left(t_{0}+45\right)=\mathrm{L}_{\mathrm{f}}\left(t_{0}+45\right)-\mathrm{L}_{\mathrm{f}}\left(t_{0}+35\right)$ and $\Delta \mathrm{L}_{\mathrm{f}}\left(t_{0}+65\right)=\mathrm{L}_{\mathrm{f}}\left(t_{0}+65\right)$ $-\mathrm{L}_{\mathrm{f}}\left(t_{0}+35\right)$ based on the latest load forecast available at $t_{0}$;

(b) The spinning reserve requirement, which we assume to be the same as it was at $t_{0}, \mathrm{RR}\left(t_{0}+45\right)$ $=\mathrm{RR}\left(t_{0}+0\right)$ and $\mathrm{RR}\left(t_{0}+65\right)=\mathrm{RR}\left(t_{0}\right)$;

(c) The interchange variation equal to the interchange schedule change as expected at $t_{0}$, $\Delta \mathrm{I}\left(t_{0}+45\right)=\mathrm{Is}\left(t_{0}+45\right)-\mathrm{Is}\left(t_{0}+35\right)$ and $\Delta \mathrm{I}\left(t_{0}+65\right)=\mathrm{Is}\left(t_{0}+65\right)-\mathrm{Is}\left(t_{0}+35\right)$, and 
(d) Generation dispatch points and other generator information from the latest market run results available at $t_{0}$.

Predicted upward generation capability: $\Delta \mathrm{P}_{\mathrm{G}}{ }^{\mathrm{up}}\left(t_{0}+45\right)=\mathrm{P}_{\mathrm{G}}{ }^{\mathrm{up}}\left(t_{0}+10\right)-\mathrm{P}_{\mathrm{G}}\left(t_{0}\right)$ and $\Delta \mathrm{P}_{\mathrm{G}}{ }^{\text {up }}$ $\left(t_{0}+65\right)=\mathrm{P}_{\mathrm{G}}{ }^{\mathrm{up}}\left(t_{0}+30\right)-\mathrm{P}_{\mathrm{G}}\left(t_{0}\right)$,

Predicted downward generation capability: $\Delta \mathrm{P}_{\mathrm{G}}{ }^{\mathrm{n}}\left(t_{0}+45\right)=\mathrm{P}_{\mathrm{G}}{ }^{\mathrm{dn}}\left(t_{0}+10\right)-\mathrm{P}_{\mathrm{G}}\left(t_{0}\right)$ and $\Delta \mathrm{P}_{\mathrm{G}}{ }^{\mathrm{dn}}$ $\left(t_{0}+65\right)=\mathrm{P}_{\mathrm{G}}{ }^{\mathrm{Wn}}\left(t_{0}+30\right)-\mathrm{P}_{\mathrm{G}}\left(t_{0}\right)$,

Thus, the predicted system upward capability can be calculated as:

$\mathrm{UP}_{\mathrm{t} 0+45}=\mathrm{Is}\left(t_{0}+35\right)+\Delta \mathrm{P}_{\mathrm{G}}{ }^{\mathrm{up}}\left(t_{0}+45\right)+\Delta \mathrm{I}\left(t_{0}+45\right)-\Delta \mathrm{L}_{\mathrm{f}}\left(t_{0}+45\right)-\mathrm{RR}\left(t_{0}+45\right)$

$\mathrm{UP}_{\mathrm{t} 0+65}=\mathrm{Is}\left(t_{0}+35\right)+\Delta \mathrm{P}_{\mathrm{G}}{ }^{\mathrm{up}}\left(t_{0}+65\right)+\Delta \mathrm{I}\left(t_{0}+65\right)-\Delta \mathrm{L}_{\mathrm{f}}\left(t_{0}+65\right)-\mathrm{RR}\left(t_{0}+65\right)$

Predicted system downward capability is:

$\mathrm{DN}_{\mathrm{t} 0+45}=\mathrm{Is}\left(t_{0}+35\right)+\Delta \mathrm{P}_{\mathrm{G}}{ }^{\mathrm{n}}\left(t_{0}+45\right)+\Delta \mathrm{I}\left(t_{0}+45\right)+\Delta \mathrm{L}_{\mathrm{f}}\left(t_{0}+45\right)$

$\mathrm{DN}_{\mathrm{t} 0+65}=\mathrm{Is}\left(t_{0}+35\right)+\Delta \mathrm{P}_{\mathrm{G}}{ }^{\mathrm{dn}}\left(t_{0}+65\right)+\Delta \mathrm{I}\left(t_{0}+65\right)+\Delta \mathrm{L}_{\mathrm{f}}\left(t_{0}+65\right)$

For the actual ramping capability, calculated for the same period, we use:

(a) The actual load change $\Delta \mathrm{La}, \Delta \mathrm{La}\left(t_{0}+45\right)=\mathrm{La}\left(t_{0}+45\right)-\mathrm{La}\left(t_{0}+35\right)$ and $\Delta \mathrm{La}\left(t_{0}+65\right)=\mathrm{La}\left(t_{0}+65\right)-$ $\mathrm{La}\left(t_{0}+35\right)$

(b) The actual spinning reserve requirement, $\mathrm{RRa}\left(t_{0}+45\right)$ and $\mathrm{RRa}\left(t_{0}+65\right)$;

(c) The actual interchange variation $\Delta \mathrm{Ia}\left(t_{0}+45\right)=\mathrm{Ia}\left(t_{0}+45\right)-\mathrm{Ia}\left(t_{0}+35\right)$ and $\Delta \mathrm{Ia}\left(t_{0}+65\right)=\mathrm{Ia}\left(t_{0}+65\right)-$ $\mathrm{Ia}\left(t_{0}+35\right)$, and

(d) Actual generation dispatch points and other generation information available at $t_{0}+65$.

Actual upward generation capability: $\Delta \mathrm{P}_{\mathrm{Ga}}{ }^{\text {up }}\left(t_{0}+45\right)=\mathrm{P}_{\mathrm{Ga}}{ }^{\text {up }}\left(t_{0}+45\right)-\mathrm{P}_{\mathrm{Ga}}\left(t_{0}+35\right)$ and $\Delta \mathrm{P}_{\mathrm{Ga}}{ }^{\text {up }}$ $\left(t_{0}+65\right)=\mathrm{P}_{\mathrm{G}}{ }^{\mathrm{up}}\left(t_{0}+65\right)-\mathrm{P}_{\mathrm{G}}\left(t_{0}+35\right)$,

Actual downward generation capability: $\Delta \mathrm{P}_{\mathrm{Ga}}{ }^{\mathrm{dn}}\left(t_{0}+45\right)=\mathrm{P}_{\mathrm{Ga}}{ }^{\mathrm{dn}}\left(t_{0}+45\right)-\mathrm{P}_{\mathrm{Ga}}\left(t_{0}+35\right)$ and $\Delta \mathrm{P}_{\mathrm{Ga}}{ }^{\mathrm{dn}}$ $\left(t_{0}+65\right)=\mathrm{P}_{\mathrm{Ga}}{ }^{\mathrm{dn}}\left(t_{0}+65\right)-\mathrm{P}_{\mathrm{Ga}}\left(t_{0}+35\right)$.

Thus, the actual system upward capability can be calculated as:

$\mathrm{UP}_{\mathrm{t} 0+45}=\mathrm{Ia}\left(t_{0}+35\right)+\Delta \mathrm{P}_{\mathrm{Ga}}{ }_{\mathrm{up}}\left(t_{0}+45\right)+\Delta \mathrm{Ia}\left(t_{0}+45\right)-\Delta \mathrm{L}_{\mathrm{a}}\left(t_{0}+45\right)-\mathrm{RRa}\left(t_{0}+45\right)$ 
$\mathrm{UP}_{\mathrm{t} 0+65}=\mathrm{Ia}\left(t_{0}+35\right)+\Delta \mathrm{P}_{\mathrm{Ga}}{ }^{\mathrm{up}}\left(t_{0}+65\right)+\Delta \mathrm{Ia}\left(t_{0}+65\right)-\Delta \mathrm{La}\left(t_{0}+65\right)-\mathrm{RRa}\left(t_{0}+65\right)$

Actual system downward capability is:

$\mathrm{DN}_{\mathrm{t} 0+45}^{*}=\mathrm{Ia}\left(t_{0}+35\right)+\Delta \mathrm{P}_{\mathrm{Ga}}{ }^{\mathrm{dn}}\left(t_{0}+45\right)+\Delta \mathrm{Ia}\left(t_{0}+45\right)+\Delta \mathrm{L}_{\mathrm{a}}\left(t_{0}+45\right)$

$\mathrm{DN}_{\mathrm{t} 0+65}^{*}=\mathrm{Ia}\left(t_{0}+35\right)+\Delta \mathrm{P}_{\mathrm{Ga}}^{\mathrm{dn}}\left(t_{0}+65\right)+\Delta \mathrm{Ia}\left(t_{0}+65\right)+\Delta \mathrm{L}_{\mathrm{a}}\left(t_{0}+65\right)$

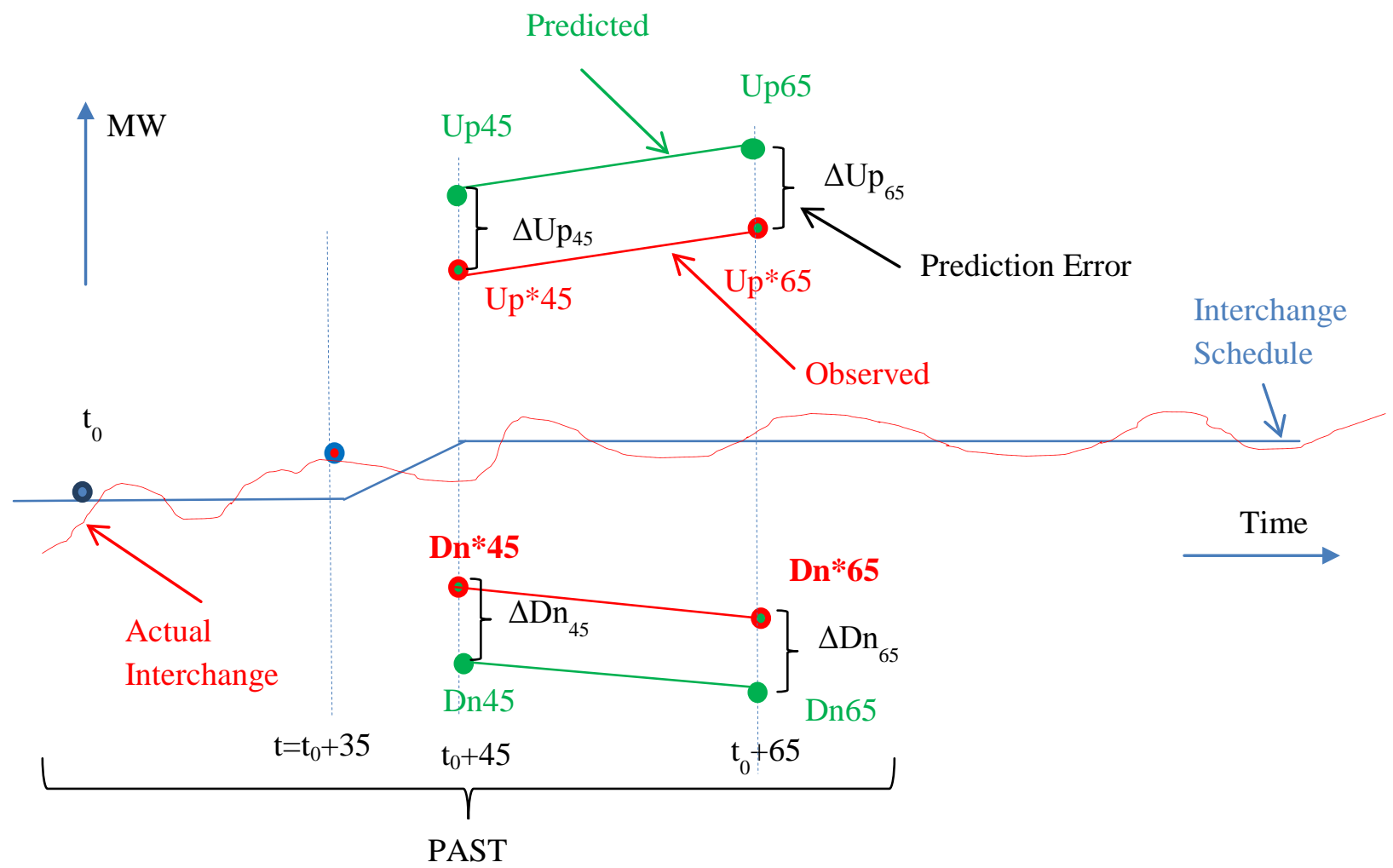

Figure 4-4. Predicted and actual upward and downward ramping capability 
Then we compare the actual upward and downward points with predicted points at $t_{0}+45$ and $t_{0}+65$ and calculate the differences between these points $\Delta \mathrm{UP}_{\mathrm{t} 0+45}, \Delta \mathrm{DN}_{\mathrm{t} 0+45}, \Delta \mathrm{UP}_{\mathrm{t} 0+65}$, and $\Delta \mathrm{DN}_{\mathrm{t} 0+65}$. We accumulate sets of these differences over the past interval T: $\left\{\Delta \mathrm{UP}_{\mathrm{t} 0+45}\right\}_{\mathrm{T}},\left\{\Delta \mathrm{DN}_{\mathrm{t} 0+45}\right\}_{\mathrm{T}},\left\{\Delta \mathrm{UP}_{\mathrm{t} 0+65}\right\}_{\mathrm{T}}$, and

$\left\{\Delta \mathrm{DN}_{\mathrm{t} 0+65}\right\}_{\mathrm{T}}$. These four sets are calculated separately for each operating hour of the day, $h=1, \ldots, 24$, and separately for the business days (BD) and weekends (WE). Finally, we will have $4 \times 24 \times 2=192$ sets of parameters $\{\Delta a b(h, d)\} \mathrm{T}$, where $a=$ "UP” or “DN", $b=$ “ $t_{0}+45$ ” or " $t_{0}+65$ ”, $h=1, \ldots, 24$, and $d=$ "BD” or "WE".

It should be noted that in these calculations, time will be changed with 5-minute increments (but not 1-hour increments). Therefore, for example, for a 3-month period T, each BD set contains about 18515 data points, and each WE set contains 7405 data points.

The next step is to build a histogram and an experimental cumulative distribution function (CDF) for each of the 192 sets. The procedure used to obtain the CDF for each set of parameter is illustrated in Figure 4-5. As a result, we obtain 192 probability density functions (PDF) characteristics, $\operatorname{PDF}\{\Delta a b$ $(h, d)\}_{\mathrm{T}}$, and $192 \mathrm{CDF}$ characteristics $\operatorname{PDF}\{\Delta a b(h, d)\}_{\mathrm{T}}$.

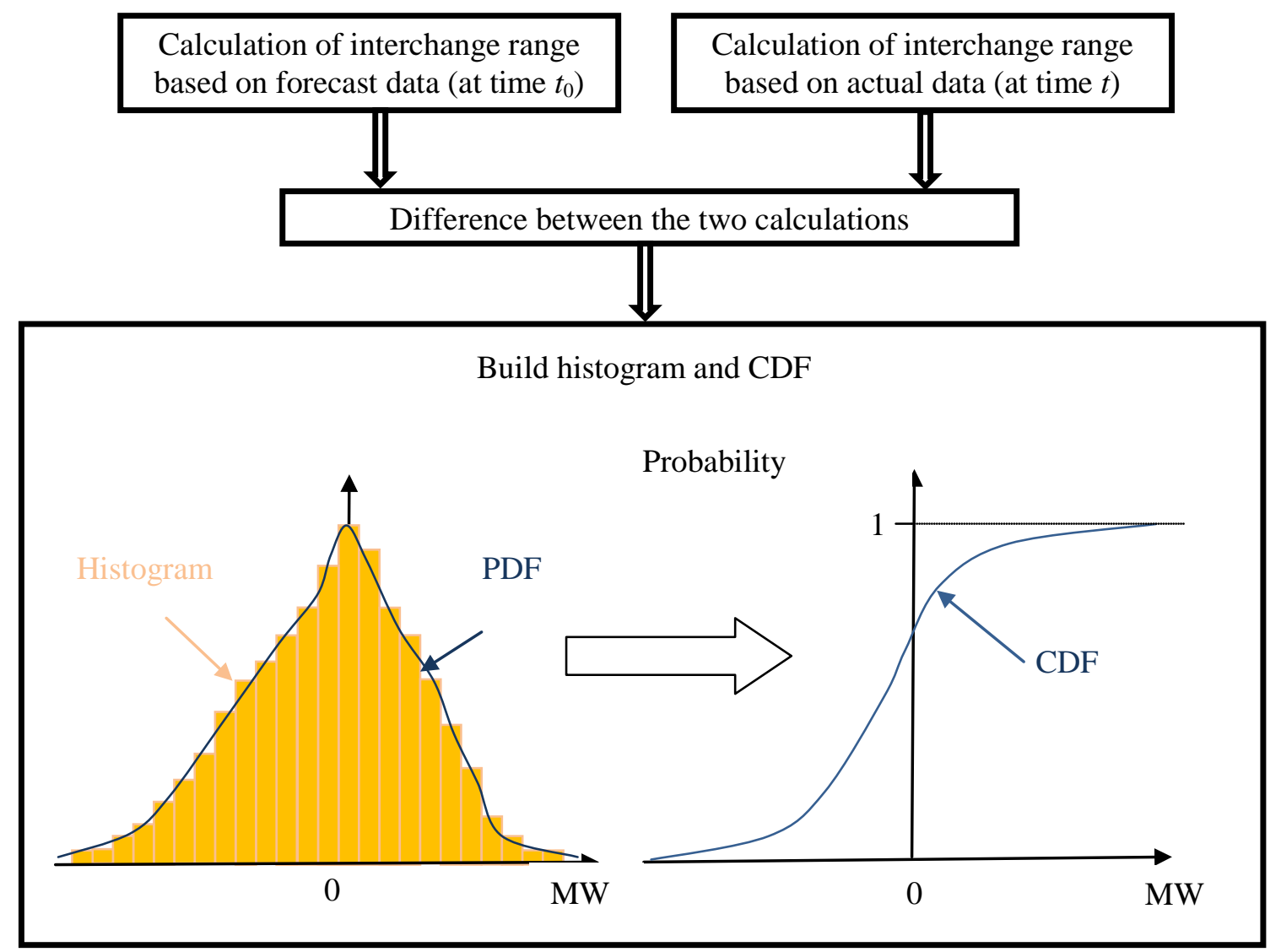

Figure 4-5. Building CDF for each variable 
Based on the statistical information together with a specified confidence level, the uncertainty of interchange range (in MW) can be quantified. For example, we can evaluate the upper and lower uncertainty bounds with 95\% confidence by cutting $5 \%$ of the points from the lower part of the UP margin's CDF, or 5\% of the points from the upper margin of the DN margin's CDF, as shown in Figure 4-6.
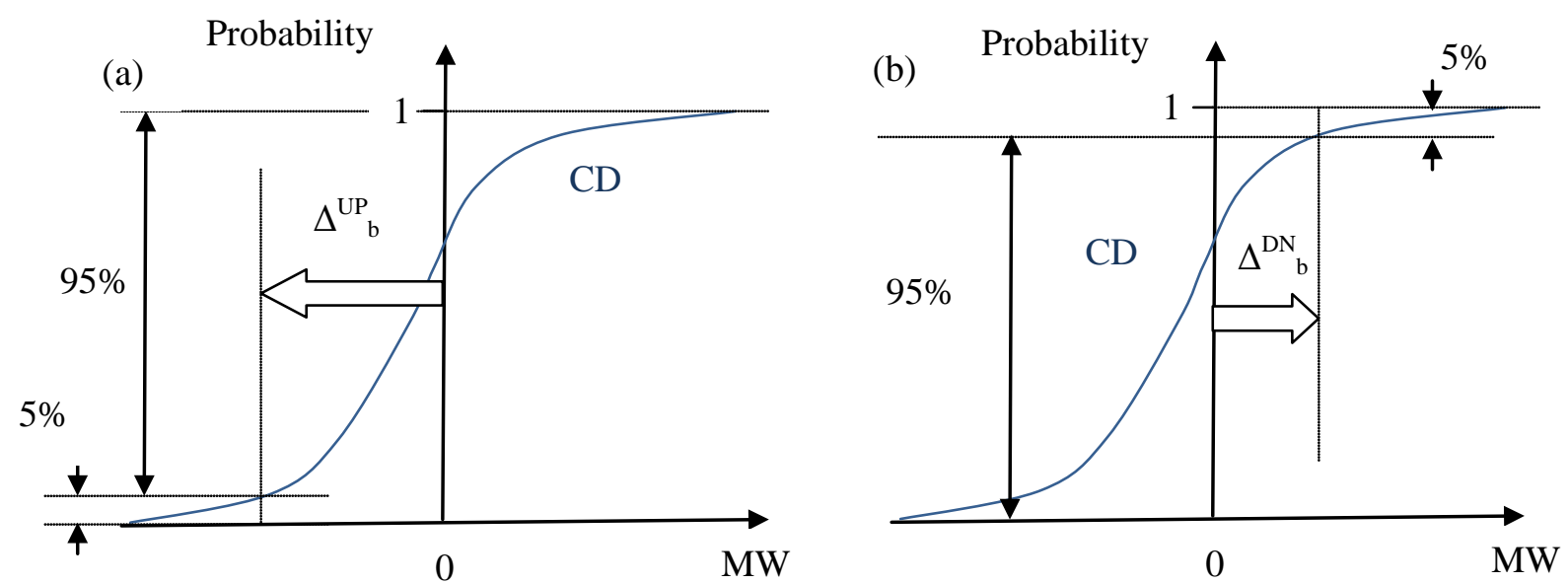

Figure 4-6. Calculation of: (a) Upward uncertainty range prediction, $\Delta^{\mathrm{UP}}$ b, and (b) Downward uncertainty range prediction, $\Delta^{\mathrm{DN}} b$

\section{3 "Look-ahead" Estimation in Real Time}

After quantifying the uncertainty for the past interval T, our remaining challenge is estimating the secure tie line interchange range in real time and ahead of time. We assume that the uncertainty ranges will remain the same as they were within the past interval $\mathrm{T}$, but specific for each operating hour of the day as well as for business days vs. weekends.

Decision on the interchange adjustments need to be made at the current time $t_{0}$, which is about $\Delta t=35$ minutes before the actual interchange adjustment starting time $t_{0}+35$, as illustrated in Figure 4-7. With today's 15-minute look-ahead dispatch, the actual operating point for each generator at $t_{0}+35$ is unknown, making it difficult to estimate the generation ramping capability. Moreover, the actual interchange and load change within the ramping duration are also unknown. 


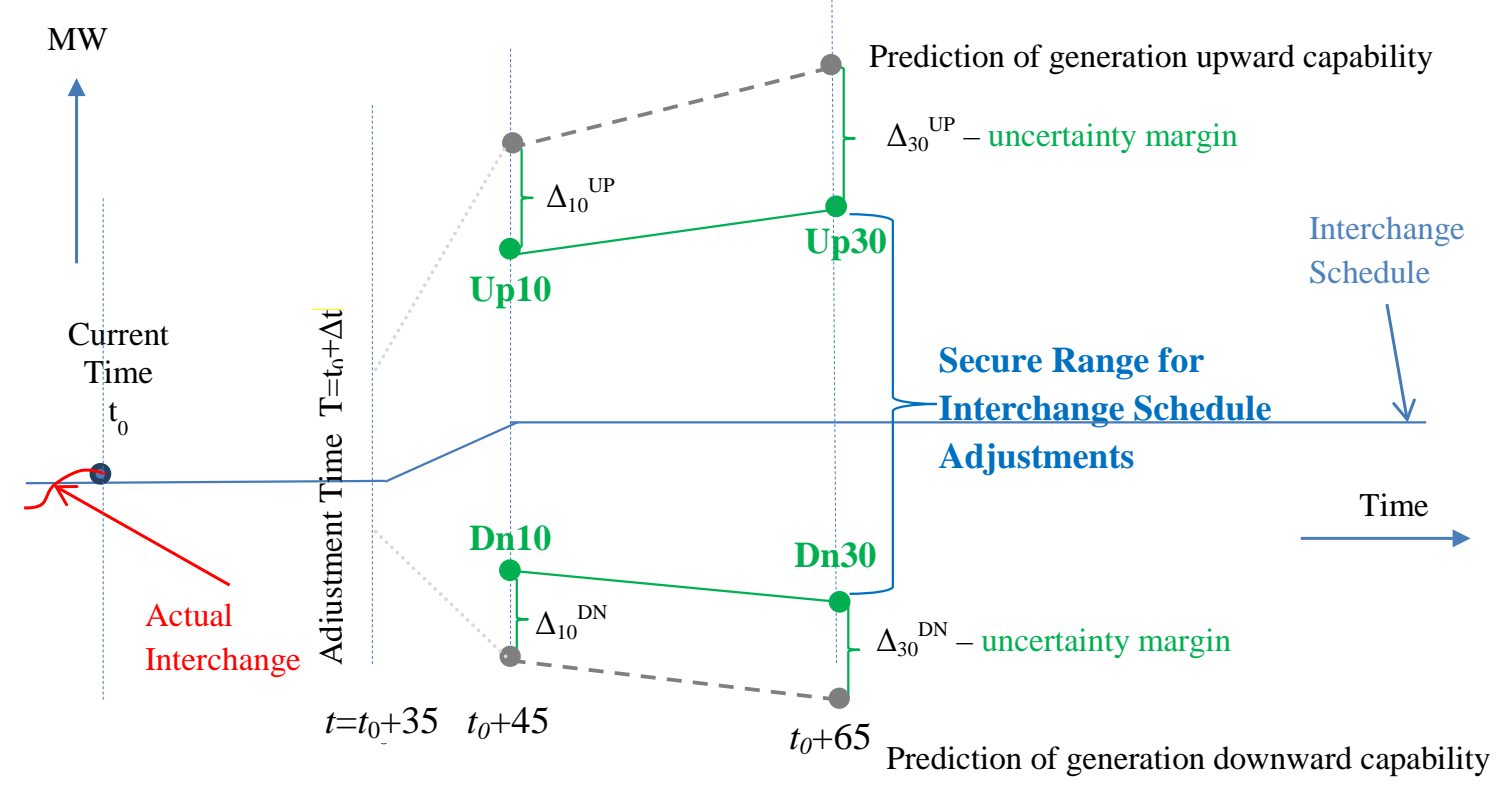

Figure 4-7. Estimation of the secure interchange adjustment range

The uncertainty of the resulting interchange adjustment range can be estimated using statistics of the historical differences between interchange ranges calculated using the actual data (at time $t_{0}+65$ ) and interchange ranges calculated using the forecast data (values predicted at time $t_{0}$ ). In this way, all sources of uncertainty discussed in this report are taken into account implicitly. The statistical information, together with the user-specified confidence level, is used to calculate the uncertainty margin, based on which the real time prediction will be adjusted. Details are provided below.

In real time, at $t_{0}$, we first predict the ramping up and ramping down capability of the system as it was described above for the past interval T. Then we simply reduce the predicted adjustment range by uncertainty margin, as shown in Figure 4-7. The margin is different for different hours of an operating day, as well as for business and weekend dates.

Finally, an example of the secure interchange adjustment range (in MW) is plotted in Figure 4-8. The black dashed lines show upward and downward generation capability predictions at time 00:50 based on the information available at the current time 00:15. The system ramping capability prediction taking into account load variation and reserve requirements is shown as the violet lines. The system capability confidence ranges (95\% confidence level) are shown using orange areas. These confidence ranges are based on the analysis of the prediction errors in the past. Thus, the secure ranges for interchange schedule adjustment at time 1:00 and 1:20 are shown by the blue points. 


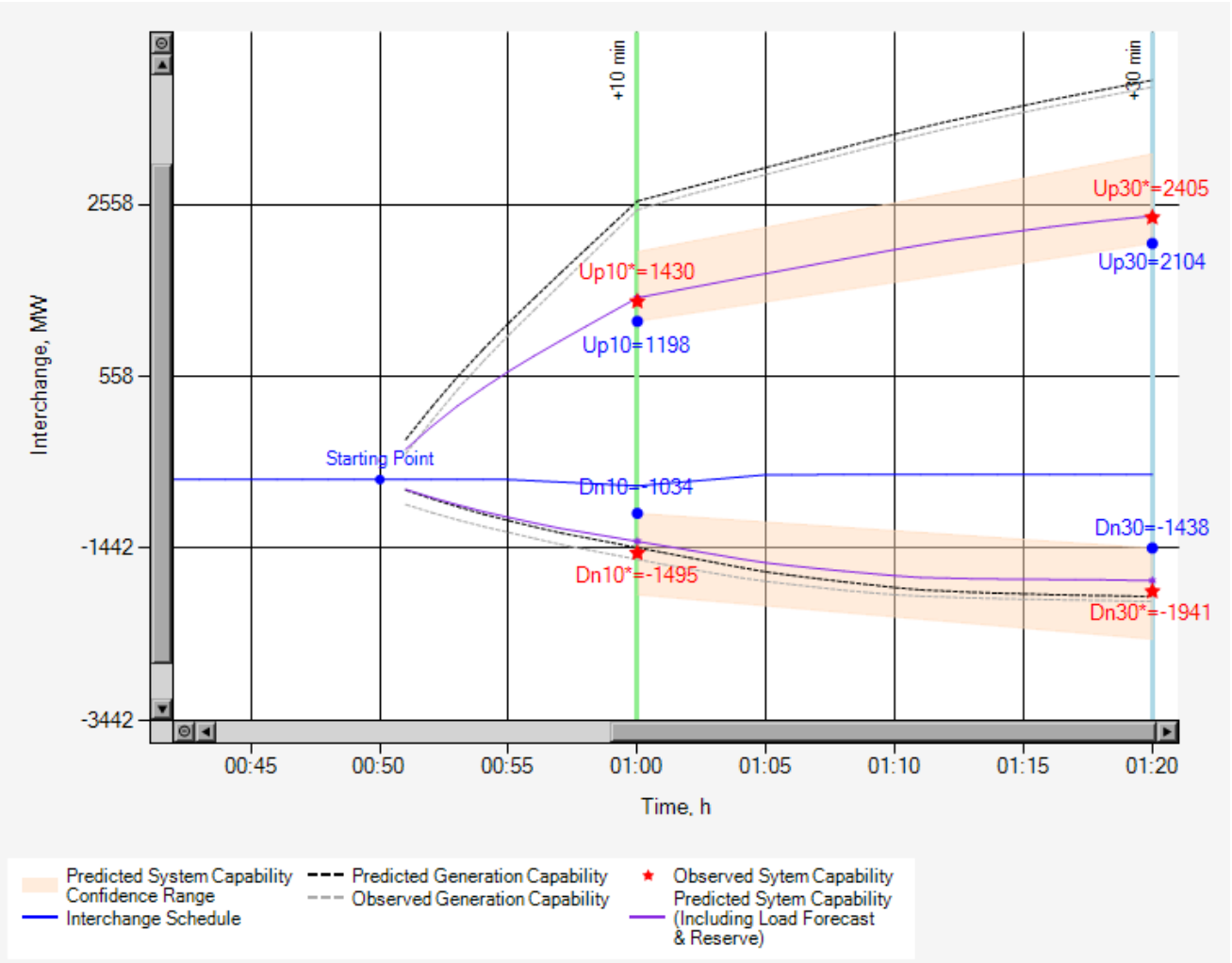

Figure 4-8. Example of actual interchange adjustment range estimation

\subsection{Validation Procedure for Results}

Validation capability is implemented in the tool, which enables system operators to evaluate the performance of the developed tool. The self-validation procedure includes comparing the actually observed system capability with predicted uncertainty ranges, and calculating the percentage of actual points found within the predicted range.

For example, the histograms of prediction errors for upward and downward capacity are plotted in Figure 4-9 and Figure 4-10, respectively. In each figure, the histograms at both $t_{0}+45$ and $t_{0}+65$ are shown.

Example simulation results are shown in Figure 4-11, where confidence level is set to be $95 \%$ for our estimation of the secure range. As can be seen from Figure 4-11, the percentages of actual points that are within the predicted interval are around 95\%, which demonstrates the precision of the proposed method.

For example, in the "Up" case, (top left of Figure 4-11), 94.79\% of the test data points were found to lie in the predicted 95\% confidence interval. 


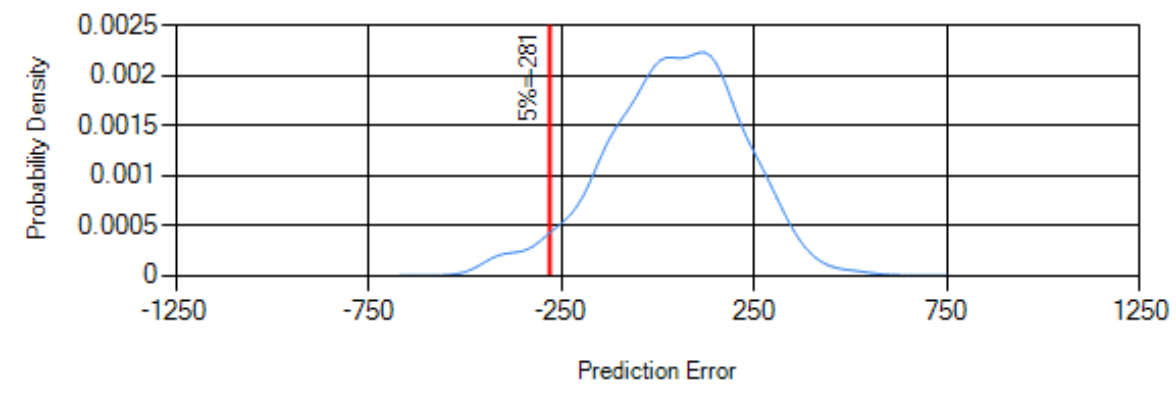

(a)

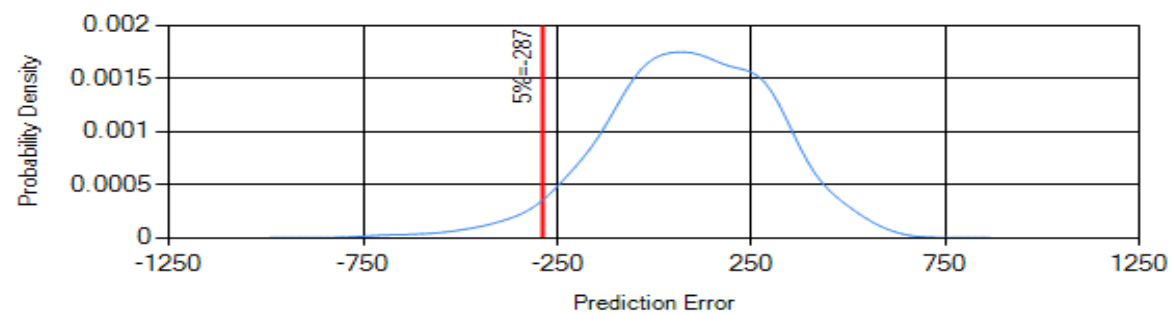

(b)

Figure 4-9. Histograms of prediction errors for upward system capability at hour ending by 1 for (a) $t_{0}+45$; (b) $t_{0}+65$

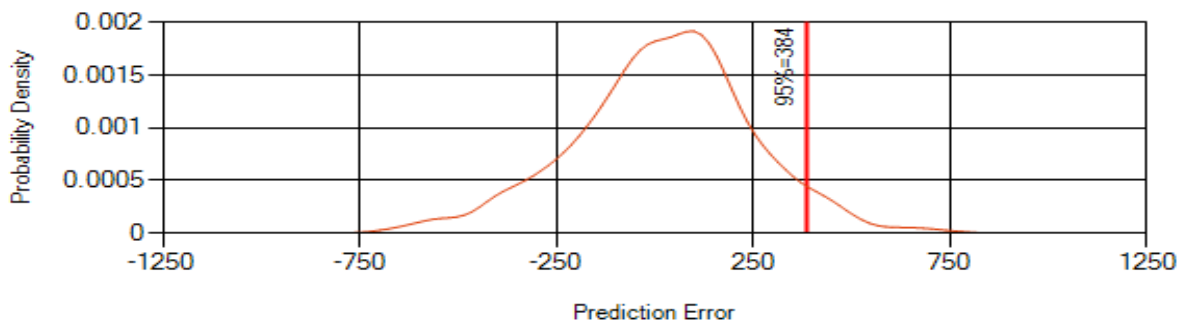

(a)

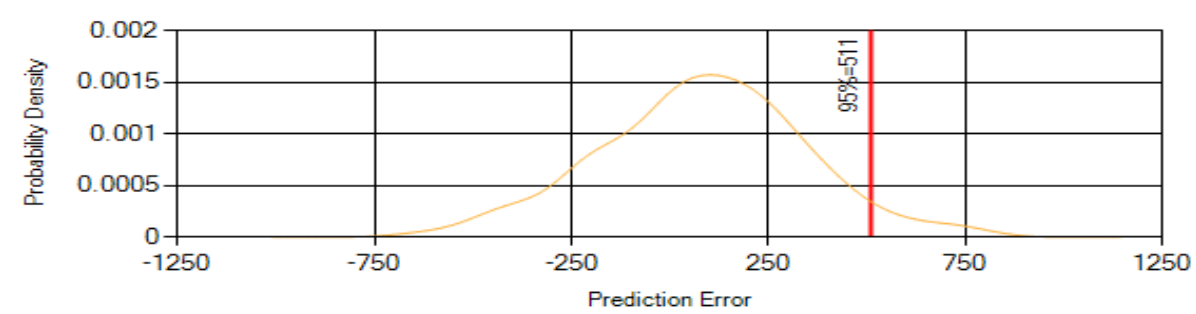

(b)

Figure 4-10. Histograms of prediction errors for downward system capability at hour ending by 1 for (a) $t_{0}+45$; (b) $t_{0}+65$

Figure 4-5 and Figure 4-6 in section 4.2 explain how the experimental histograms provided in Figure 4-9 and Figure 4-10 are further used to quantify uncertainties in the upward and downward directions with certain user-specified level of confidence.

The probability distributions in Figure 4-9 and Figure 4-10 have the following statistical characteristics - see. 
Table 4-1. Statistical characteristics of prediction errors for the upward and downward interchange adjustment capability

\begin{tabular}{|l|l|l|l|l|}
\hline Parameter & Up45 & Up65 & Dn45 & Dn65 \\
\hline Mean (MW) & 40 & 90 & 20 & 82 \\
\hline STD $(\mathrm{MW})$ & 172 & 211 & 225 & 261 \\
\hline
\end{tabular}

In Table 4-1, “Up45” and "Up65” stand for the upward interchange adjustment capability for the moments $t_{0}+45$; and $t_{0}+65$, and "Dn45" and "Dn65" denote for the downward interchange adjustment capability for the moments $t_{0}+45$; and $t_{0}+65$, correspondingly.

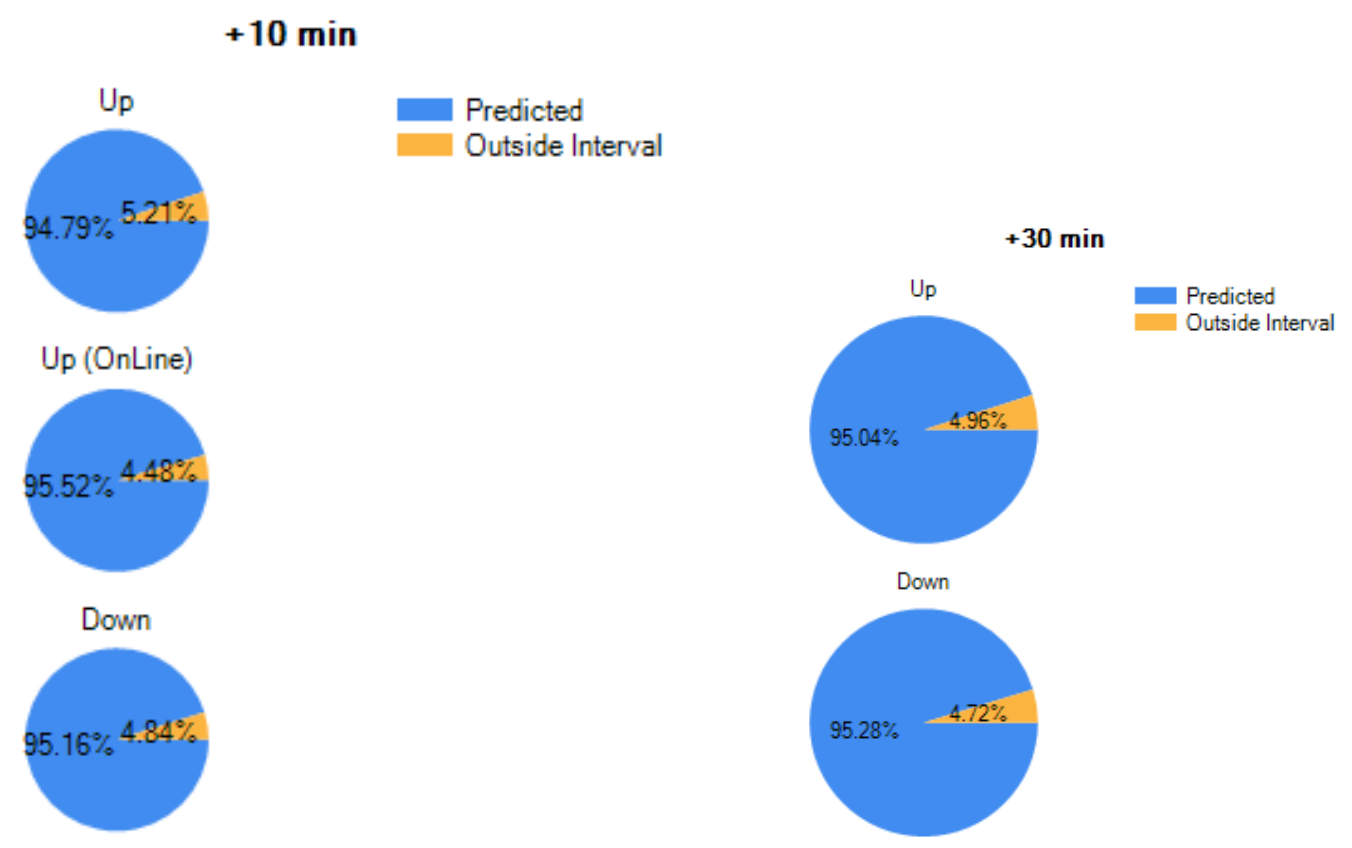

Figure 4-11. Self-validation results. 



\subsection{DINA Tool Description}

A tool for quantifying secure ranges for intra-hour interchange adjustments and related uncertainty has been implemented by PNNL based on the methodology developed in this project and outlined above in this report. In this section, a description of the developed software is presented. Brief portrayals of software requirements, its structure, main features, and graphical user interface (GUI) are given below.

\subsection{Software Requirements and Structure}

Microsoft Visual Studio ${ }^{\circledR} 2010$ was used to develop the software tool using the Microsoft ${ }^{\circledR}$.NET Framework 4 for the Microsoft Windows ${ }^{\circledR}$ platform. The tool consists of three tiers: the database, the generation capability and uncertainty evaluation dynamic-link library (DLL) and the GUI - see Figure 5-1. The DINA tool uses Oracle ${ }^{\circledR} 11 \mathrm{~g}$ for database functionality. The uncertainty evaluation DLL contains classes providing capabilities to interact with the database, calculate generation ramping capability, and estimate secure ranges for interchange adjustments. Based on user input, the uncertainty GUI calls the DLL to calculate required outputs and then display the results in graphical and tabular forms.

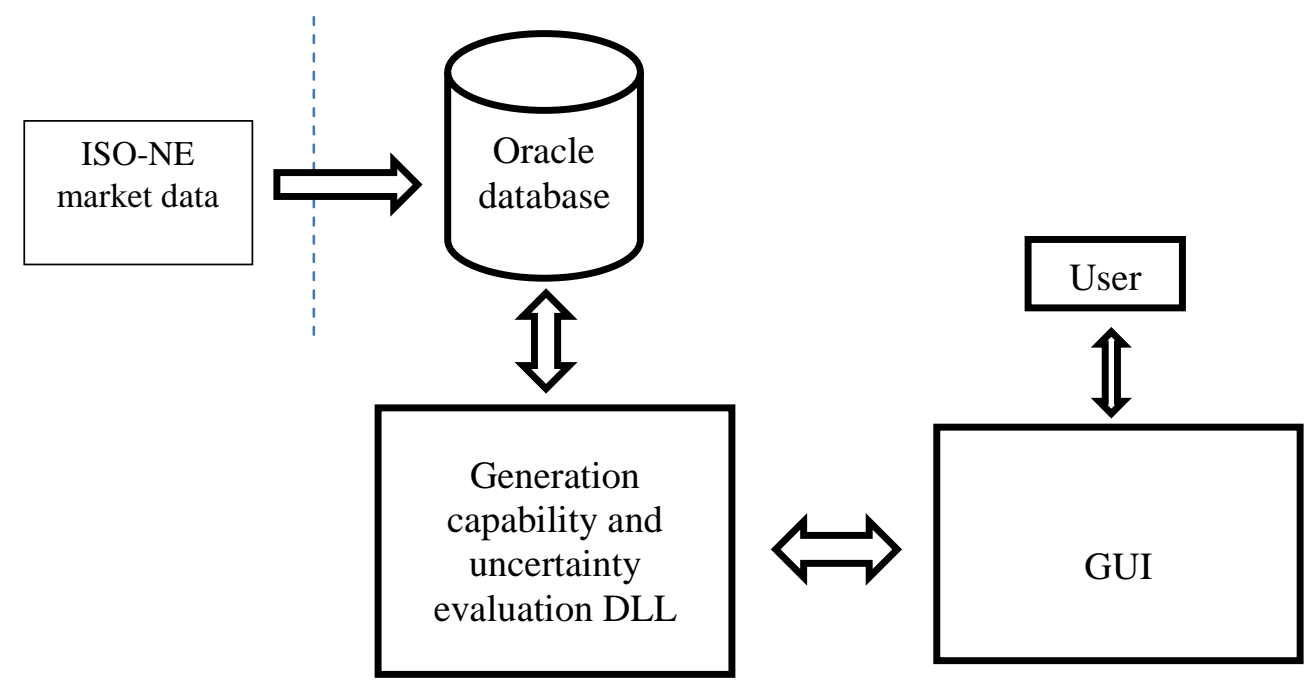

Figure 5-1. The software structure

\subsection{Graphical User Interface}

The software tool GUI has several operating screens. The first screen is the main display (“Actual Screen") that shows the actual and forecasted information as well as interchange requirements quantifications - see Figure 5-2. Three other screens (“Generation”, "Prediction Errors” and "Statistical Analysis”) provide interactive visualizations for system generation ramping capability, interchange prediction statistics, load forecast and interchange errors statistics, correspondingly. 


\subsubsection{Main Display}

Figure 5-2 shows a screenshot of the main display - “Actual Screen”. The user can select the real time mode or any specific date and time in the past to review information on forecasted and actual parameters in tabular or graphical form. The user selects which of the following should be plotted

- Load forecast,

- Generation requirements forecast (= Load forecast + Interchange schedule),

- Generation schedule,

- Actual load,

- Actual generation,

- Actual generation requirement (= Actual load + Actual interchange),

- Interchange schedule,

- Actual interchange,

- Interchange schedule requirements (= Generation schedule - Load forecast),

- Actual interchange requirements (= Actual Generation - Actual load),

- Generation ramping capability (available interchange adjustment range), including the following information and influencing factors:

o Predicted total generation ramping capability with and without fast starting units,

o Predicted total ramping capability with the load forecast (LF) change accounted for,

o Predicted total ramping capability with the load forecast (LF) change and reserve requirements accounted for,

o Actually observed ramping capability (for the past dispatch intervals only), and

- User-specified confidence level for uncertain factors involved in the analysis.

The user also selects dates for the analysis, including operating hours in the past.

The tool then queries the database containing ISO-NE market information, processes this information, and generates the plots based on user-specified options listed above. 


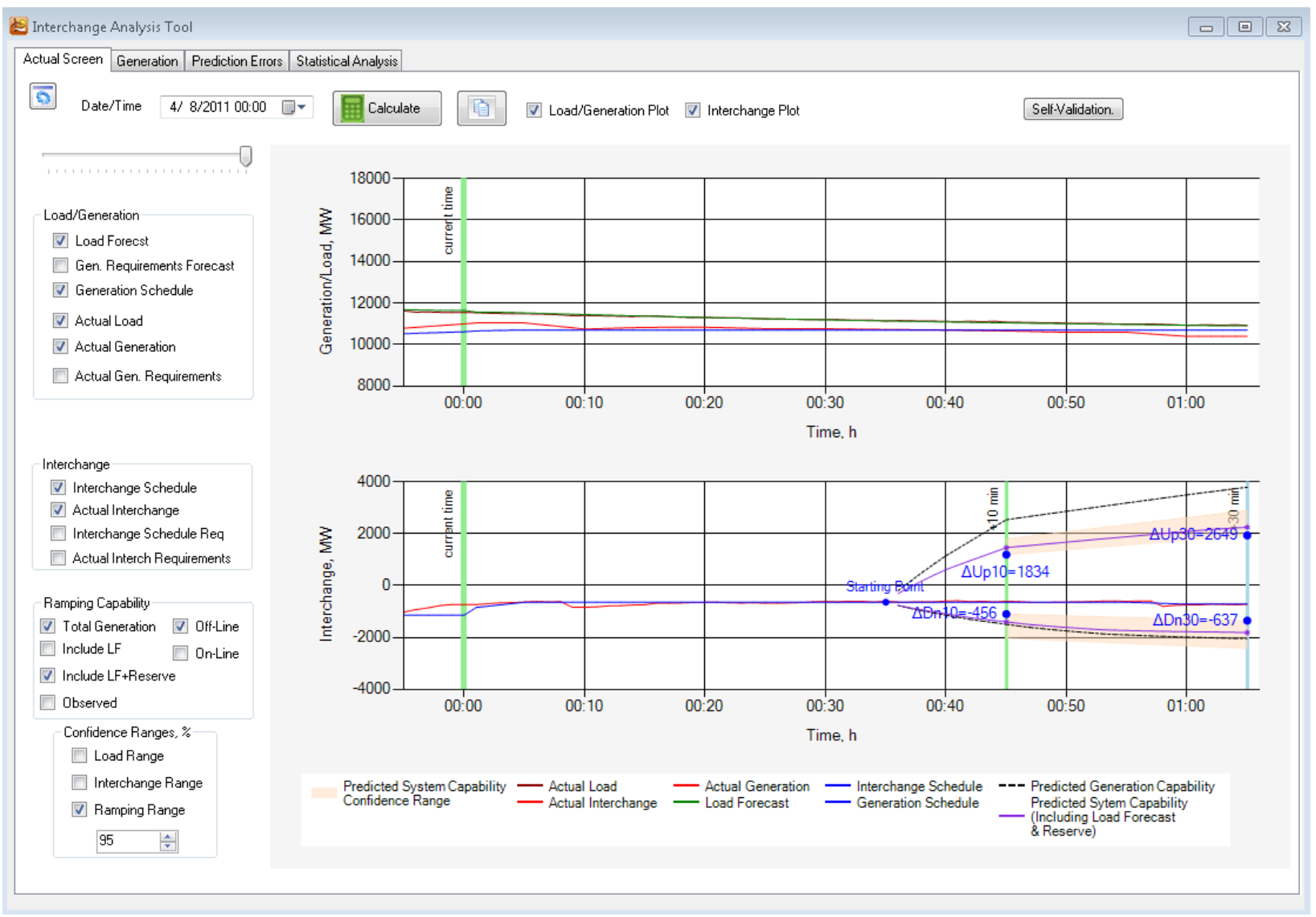

Figure 5-2. The main GUI

\subsubsection{Generation Ramping Capability Screen}

Generation ramping capability screen is presented in Figure 5-3. Generation information based on the latest market case is shown on this screen. This information includes the status of the units, actual generation, desired dispatch point (DDP), and unit maximum (Pmax) and minimum (Pmin) generation limits. Calculated generation ramping capability limits for each unit, as well as total generation capability limits, are also displayed. 


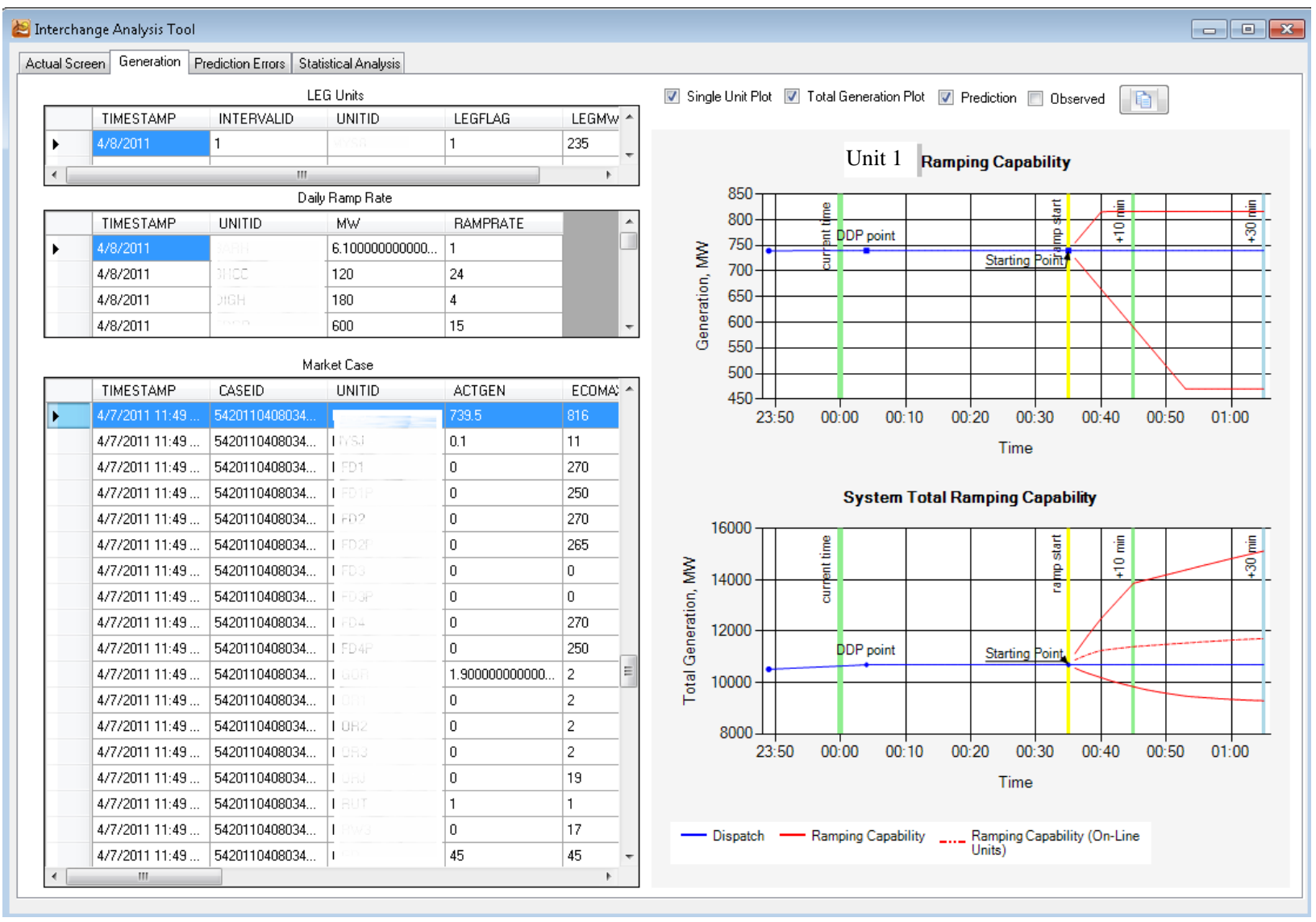

Figure 5-3. Generation ramping capability screen

Maximum ramping capability characteristics of online dispatchable unit (Unit 1) is shown in Figure 5 -3. The "current time" $t_{0}$ is 12:00 a.m. The unit dispatch instruction is shown by the blue line. The beginning of the blue line is actual generation from the latest market case. DDP point is the next desired dispatch point (= market approval time +15 min). Beyond 15 minutes after the market run, dispatch information is not available; therefore, it is assumed that the unit will stay at the DDP point. For the current time used in this example, the unit can start moving at starting point 00:35 with the maximum allowable ramp rate. The ramping characteristic for upward and downward generation is shown by the red curves. The unit ramping characteristic is also limited by the maximum $\left(\mathrm{EcoMax}^{1}\right)$ and minimum $\left(\right.$ EcoMin $\left.^{2}\right)$ generation.

The total generation capability, also shown in in Figure 5-3, is calculated as a sum of ramping characteristics of all available units. The maximum ramping capability for the whole system is shown by

\footnotetext{
${ }^{1}$ Economic Maximum (EcoMax) - The highest unrestricted level of electric energy (MW) a resource is able to generate, representing the highest megawatt output available from the resource for economic dispatch [ISO-NE Glossary].

${ }^{2}$ Economic Minimum (EcoMin) - The minimum amount of electric energy (MW) that a generating resource must be allowed to produce while under economic dispatch. A generator may request to self-schedule and increase its offered Economic Minimum to the desired level of MW [ISO-NE Glossary].
} 
the red curve in Figure 6-4. The dashed red line represents total generation ramping capability for online units only (when the fast-start offline units are not considered).

More details and examples on the generation ramping capability screen can be found in section 6 .

\subsubsection{Interchange Limits Prediction Errors Statistics Screen}

Information on prediction errors for the maximum upward and downward interchange adjustment limits observed in the past is presented on the screen in Figure 5-4. Performed analysis is conducted for a user-specified time interval (e.g., 30 days). The interchange adjustment limits statistics provides information on 10- and 30-minute ahead prediction errors, including their mean value, standard deviation (STD), minimum and maximum values. These parameters are calculated separately for the 10-minute ahead upward limit prediction error (ErrorUp10), 10-minute ahead downward limit prediction error (ErrorDn10), 30-minute ahead upward limit prediction error (ErrorUp30), and 30-minute ahead downward limit prediction error (ErrorDn30). Additionally, based on ISO-NE request, the tool calculates statistical characteristics of 10-minute ahead upward limit prediction errors for online units only, without considering the fast start up units that may be available (ErrorUp10OnLine).

PDFs of prediction errors and essential statistical information, including mean value, standard deviation, $\mathrm{min} / \mathrm{max}$ values, are also displayed. Percentiles corresponding to the $95 \%$ confidence level are shown by red vertical lines. A detailed explanation on how the uncertainty intervals are calculated based on the user-specified confidence level is provided in section 4.2.

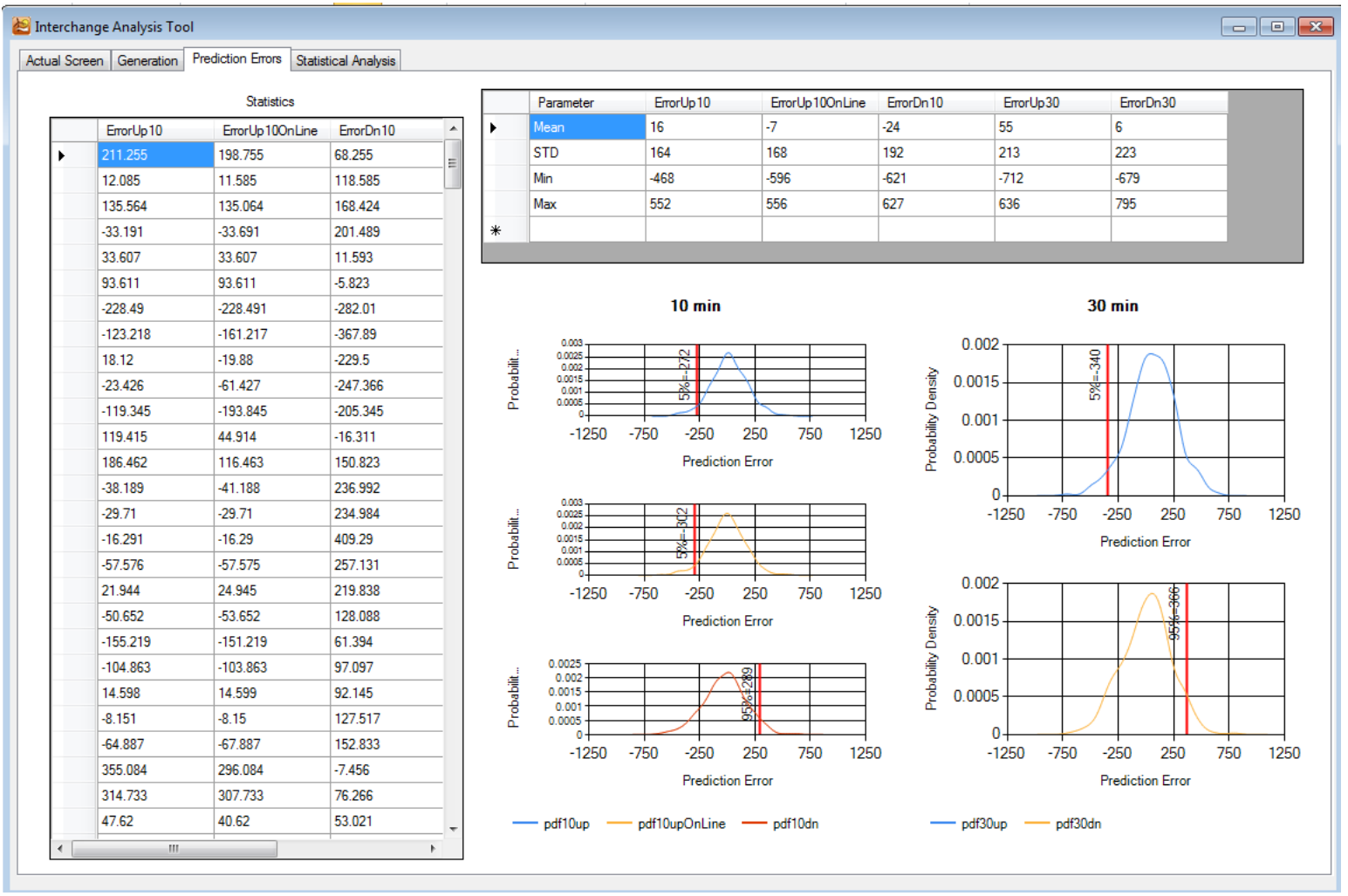

Figure 5-4. Prediction errors statistic screen 
More information on the interchange limits prediction errors information is provided in section 6 .

\subsubsection{Load Forecast, Interchange and Generation Errors Statistics Screen}

Statistics on load forecast errors as well as interchange and generation deviations from their schedules are shown on the statistics screen - see Figure 5-5. The tool displays this information for a time interval $\mathrm{T}$, restricted by the user-specified beginning and ending time. The user can also select which plots he or she wants to see on this screen, i.e. the parameter plot, the error plot or both plots as shown in Figure 55. Information on the following parameters can be displayed:

- Load forecast for $5,10, \ldots$, and 60 minutes ahead,

- Actual load,

- Interchange schedule.

- Actual interchange.

- Generation schedule calculated as a sum of online generators’ desired dispatch points (DDPs) provided in the market database for 15 minutes after the market run.

- Actual generation taken from two sources: from the ISO-NE plant information (PI) historian, and from the market database. This information can be displayed but is not used in the analysis because of the difficulties with assembling complete and accurate information on the actual generation from all online generators.

- Generation requirement forecast (can be displayed, but not included in the scope of this work), and

- Actual generation requirement (can be displayed, but not included in the scope of this work).

The user can select the error information formation to be displayed in this screen:

- Load forecast error (LF).This is the difference between load forecast for $5,10, \ldots$, or 60 minutes ahead, and actual load.

- Interchange error (= deviation) (Int). This is the difference between actual interchange and interchange schedule. The dependence of interchange error on look-ahead time is not detected nor reflected in this screen.

- Total uninstructed deviation of ISO-NE generators (Gen). This is the difference between actual generation and its market-based schedule. The dependence of uninstructed deviations on look-ahead time is not detected nor reflected in this screen. This information can be displayed but is not used in the analysis because of the difficulties with assembling complete and accurate information on the actual generation of all online generators. 
PDFs, and CDFs of the load forecast (LF) errors and interchange deviations can be also calculated and displayed. Analysis can be done for the entire interval T (see PDF and CDF functions) or for a userspecified operating hour, h, of the day (see PDF(h) and $\mathrm{CDF}(\mathrm{h})$ functions). Load forecast errors can be analyzed for different look-ahead intervals, e.g., 5, 10,..., 60 minutes ahead.

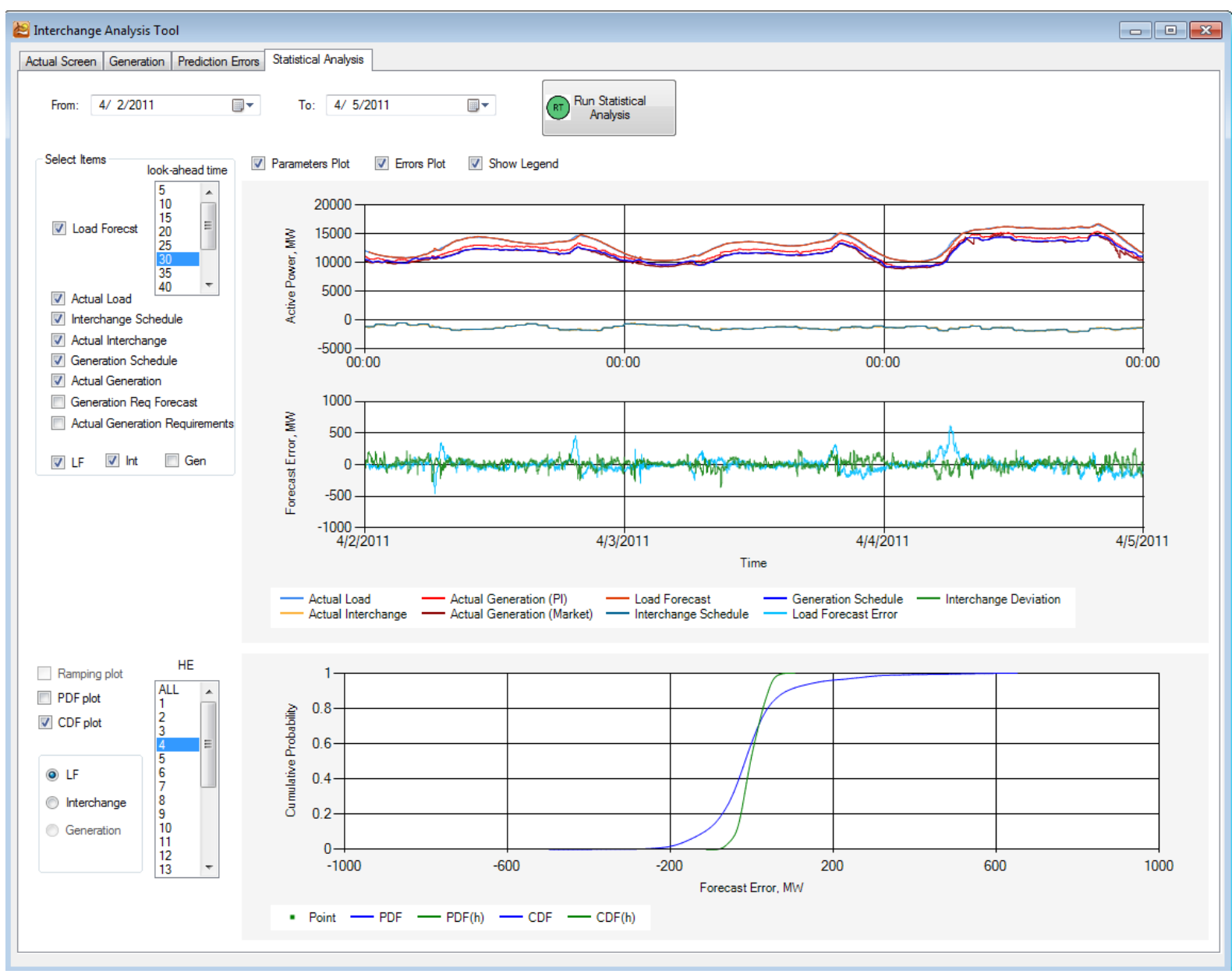

Figure 5-5. Load forecast, interchange and generation schedule errors statistics 



\subsection{Capabilities of the DINA Tool and its Validation}

Using the DINA tool, we conducted case studies to validate the approach and to provide more information on the capabilities of the tool. The case study used real data from ISO-NE. The dataset included: market information on generation characteristics and dispatch, actual load, load forecast, actual interchange, interchange schedule, and actual generation.

Simulations were performed based on the ISO-NE operational dataset for the year 2011. To illustrate the generation ramping capability and interchange secure adjustment range prediction functionalities of the DINA tool in this report, the case of May 1, 2011, hour ending by 11:00 a.m., is used as an example in sections 6.1 and 6.2. Sixty days of information before May 1, 2011, were processed to collect statistics making the prediction possible. Results of predictions are compared against the actual characteristics for the analyzed operating hour.

In section 6.3, self-validation results are provided. The self-validation functionality was run for a test window containing one month of information. Results were compared with what actually happened within this month.

All graphical illustrations in this section were provided by the developed DINA tool.

\subsection{Generation Ramping Capability}

To calculate available generation ramping capability for May 1, 2011, hour ending by 11 a.m., the latest market information available at 10 a.m. was used. Market case includes information on all generation units to be available for 15 minutes after the market approval time. For the "current time" $t_{0}$ (5/1/2011, 10:00 a.m.), the latest market case was approved at 5/1/2011 9:49 a.m.

Table 6-1 shows an example of the market information. It includes a timestamp, identification (ID) of the market case, unit name, actual generation from the latest state estimation run, maximum and minimum generation limits, unit ramp rate, fast start status, unit control mode (UCM) ${ }^{1}$, claimed generation achievable in 10 and 30 minutes for fast start units, and unit minimum run and down time. In total, there are more than two hundred units in the system in the market case.

\footnotetext{
${ }^{1}$ Unit control mode (UCM):

- $\quad \mathrm{UCM}=1$ - offline unit, not available

- $=2-$ offline/available

- $=3-$ online, non-dispatchable

- $=4$ online, dispatchable

- $\quad=5$ - not used

- $=6-$ regulating unit
} 
Table 6-1. Market case

\begin{tabular}{|c|c|c|c|c|c|c|c|c|c|c|c|}
\hline Date and Time & Case ID & $\begin{array}{l}\text { Unit } \\
\text { Name }\end{array}$ & $\begin{array}{c}\text { Actual } \\
\text { Gen }\end{array}$ & EcoMa & EcoMin & $\begin{array}{c}\text { Ramp } \\
\text { Rate }\end{array}$ & DDP & $\begin{array}{l}\text { Fast } \\
\text { start }\end{array}$ & UCM & $\begin{array}{c}\text { Claim } \\
10^{1}\end{array}$ & Claim $30^{2}$ \\
\hline & & & MW & MW & MW & $\mathrm{MW} / \mathrm{min}$ & MW & --- & --- & MW & MW \\
\hline 5/1/2011 9:49 AM & 1420110501134950 & Unit 1 & 149.5 & 157 & 110 & 5 & 150 & 1 & 4 & 0 & 0 \\
\hline 5/1/2011 9:49 AM & 1420110501134950 & Unit 2 & 0 & 98 & 98 & 8 & 0 & 1 & 2 & 0 & 98 \\
\hline & & & & & & & & & & & \\
\hline 5/1/2011 9:49 AM & 1420110501134950 & Unit $\mathrm{N}$ & 2.6 & 3 & 3 & 0.4 & 3 & 0 & 3 & 0 & 0 \\
\hline
\end{tabular}

Based on the market information, ramping capability characteristic of each unit is calculated. Maximum ramping capability of an online dispatchable unit is shown in Figure 6-1. The "current time" $t_{0}$ is 10:00 a.m. The unit dispatch instruction is shown by the blue line. The beginning of the blue line is actual generation from the latest market case. DDP point is the next desired dispatch point (market approval time $+15 \mathrm{~min}$ ). Beyond 15 minutes, dispatch information is not available; therefore, it is assumed that the unit will stay at the DDP point. The unit starts moving (ramping starting point) at 10:35 a.m. with the maximum allowable ramp rate. The ramping characteristic for upward and downward generation is shown by the red curves. The unit ramping characteristic is also limited by the maximum $\left(\right.$ EcoMax $\left.^{3}\right)$ and minimum $\left(\right.$ EcoMin $\left.^{4}\right)$ generation.

${ }^{1}$ Claim 10 - The generation output level, expressed in megawatts, that a generator can reach from an off-line state within 10 minutes after receiving a dispatch instruction from ISO. This value is required as part of the generator's offer data and is used by ISO New England to evaluate the generator's contribution towards meeting operating- and replacement-reserve requirements [ISO-NE Glossary].

${ }^{2}$ Claim 30 - The generation output level, expressed in megawatts, that a generator can reach from an off-line state in 30 minutes after receiving a dispatch instruction from ISO. This value is required as part of the generator's offer data and is used by ISO New England to evaluate the generator's contribution towards meeting the New England Control Area's operating and replacement reserve requirements [ISO-NE Glossary].

${ }^{3}$ Economic Maximum (EcoMax) - The highest unrestricted level of electric energy (MW) a resource is able to generate, representing the highest megawatt output available from the resource for economic dispatch [ISO-NE Glossary].

${ }^{4}$ Economic Minimum (EcoMin) - The minimum amount of electric energy (MW) that a generating resource must be allowed to produce while under economic dispatch. A generator may request to self-schedule and increase its offered Economic Minimum to the desired level of MW [ISO-NE Glossary]. 


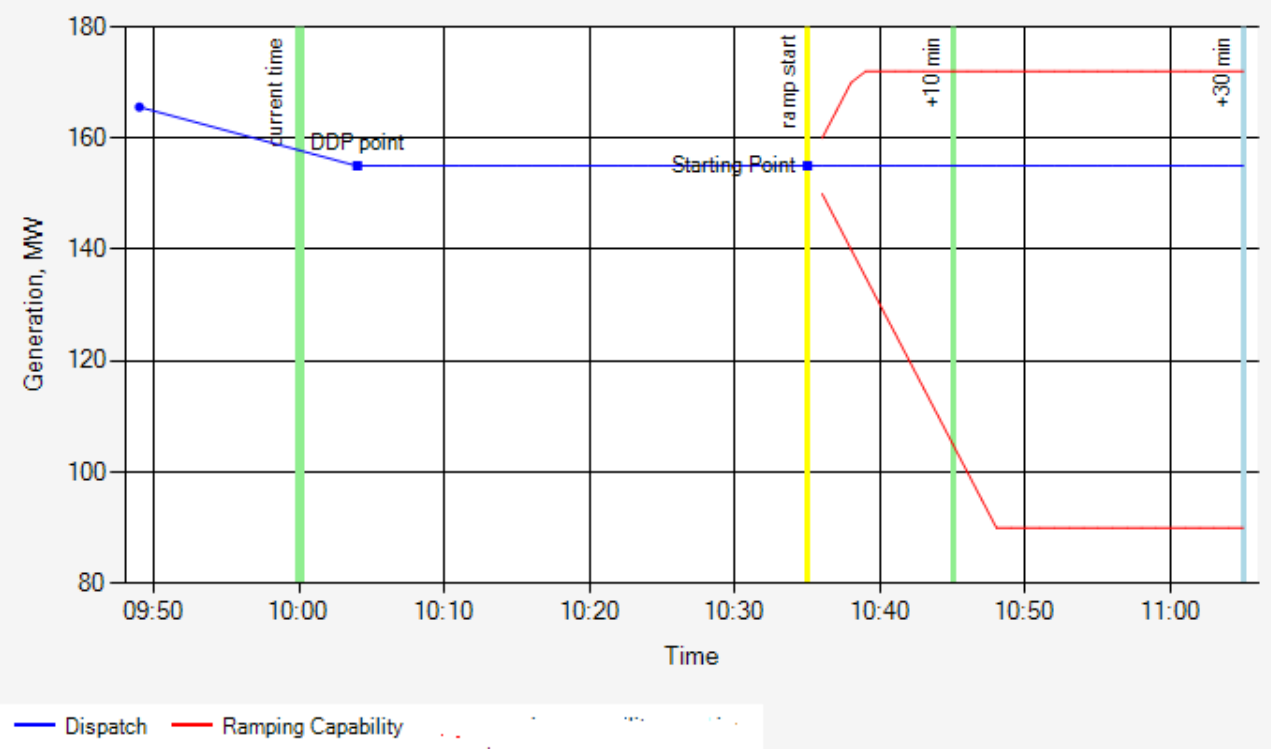

Figure 6-1. Ramping characteristic of online dispatchable unit.

Offline fast-start units are also considered in the proposed methodology. Fast-start units must achieve their claimed generation level provided in the market case within 10 and 30 minutes after the start. Figure 6-2 shows an example of ramping characteristic of an offline fast-start unit. Generation at the starting point is zero (when the unit is offline). Then the unit starts moving linearly and achieves the "Claim10" point in 10 minutes and the "Claim30" within 30 minutes (see red curve in Figure 6-2)

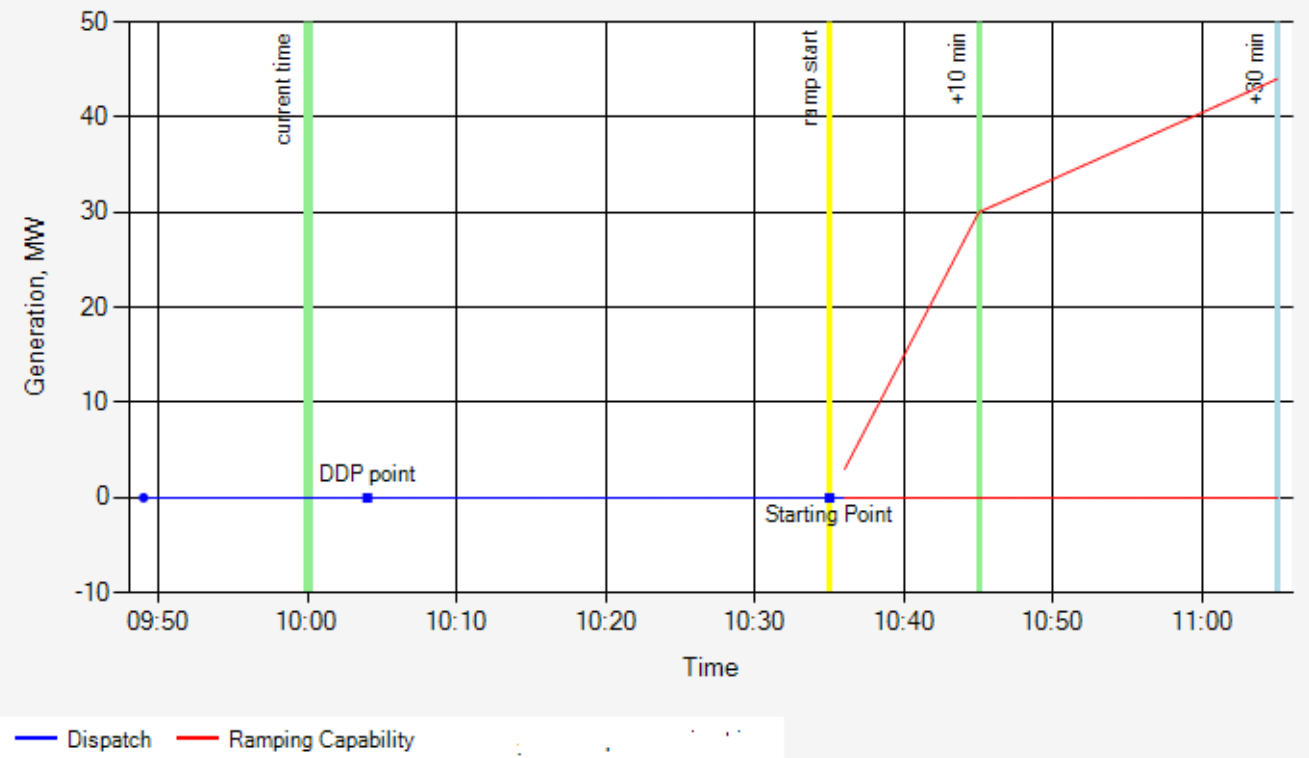

Figure 6-2. Ramping characteristic of an offline fast-start unit

There is also a special case when generation units have segmented ramp rate characteristic. An example of segmented ramp data is given in Table 6-2. The maximum ramping capability characteristic for this unit is presented in Figure 6-3. One can see that at the downward generation capability (red curve) 
has a ramp rate of $1 \mathrm{MW} / \mathrm{min}$ from the starting point (250 MW) to the generation equal to $235 \mathrm{MW}$. After the $235 \mathrm{MW}$ point, the downward ramp rate is $5 \mathrm{MW} / \mathrm{min}$. The unit does not have upward ramping capability because it is limited by unit's EcoMax parameter.

Table 6-2. Ramp segments data

\begin{tabular}{|c|l|c|c|}
\hline Date & Unit Name & Segments & Ramp \\
\hline & & MW & MW/min \\
\hline $5 / 1 / 2011$ & Unit 1 & 1 & 5 \\
\hline $5 / 1 / 2011$ & Unit 1 & 235 & 5 \\
\hline $5 / 1 / 2011$ & Unit 1 & 236 & 1 \\
\hline $5 / 1 / 2011$ & Unit 1 & 250 & 1 \\
\hline $5 / 1 / 2011$ & Unit 1 & 251 & 1 \\
\hline $5 / 1 / 2011$ & Unit 1 & 275 & 1 \\
\hline
\end{tabular}

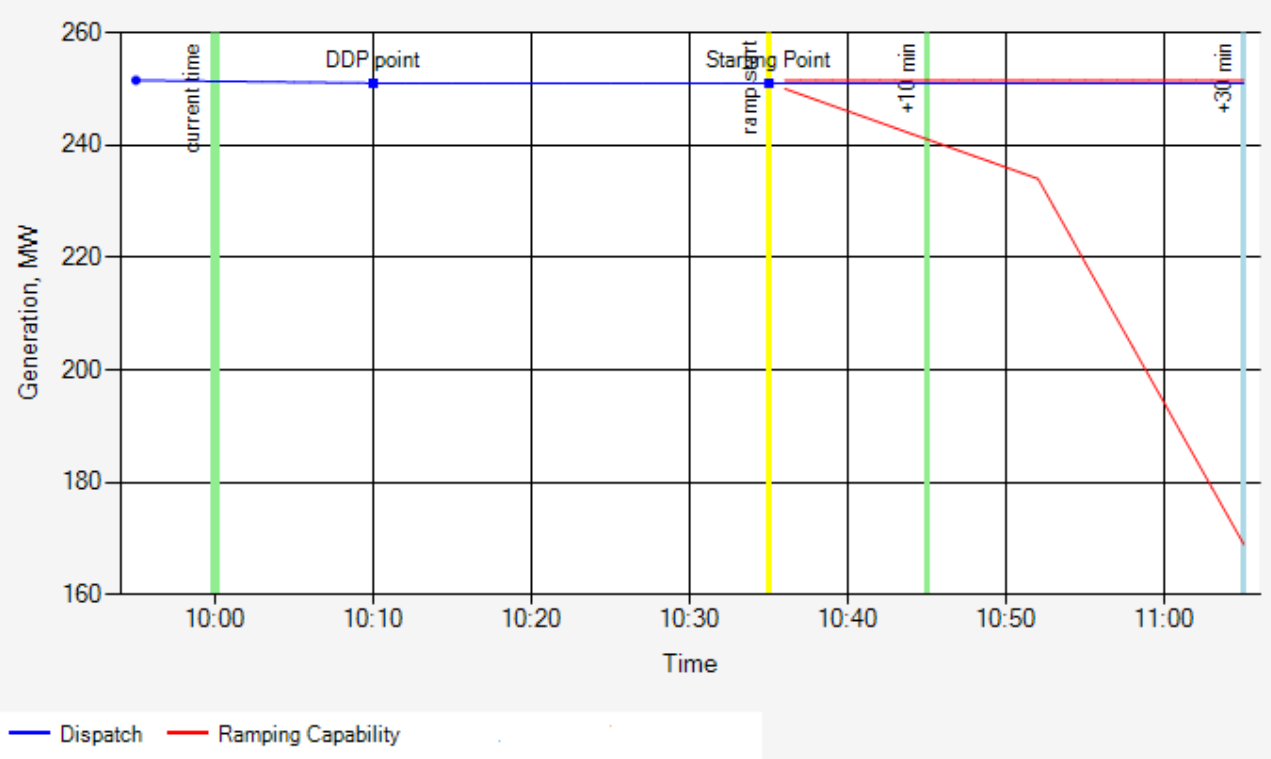

Figure 6-3. Ramping characteristic of the unit with ramp segments

After calculation of the individual ramping characteristics of each unit, the total generation capability was calculated as a sum of ramping characteristics of all available units. The maximum ramping capability for the whole system is shown by the red curve in Figure 6-4. The dashed red line represents total system ramping capability for online units only when the fast-start offline units are not considered. 


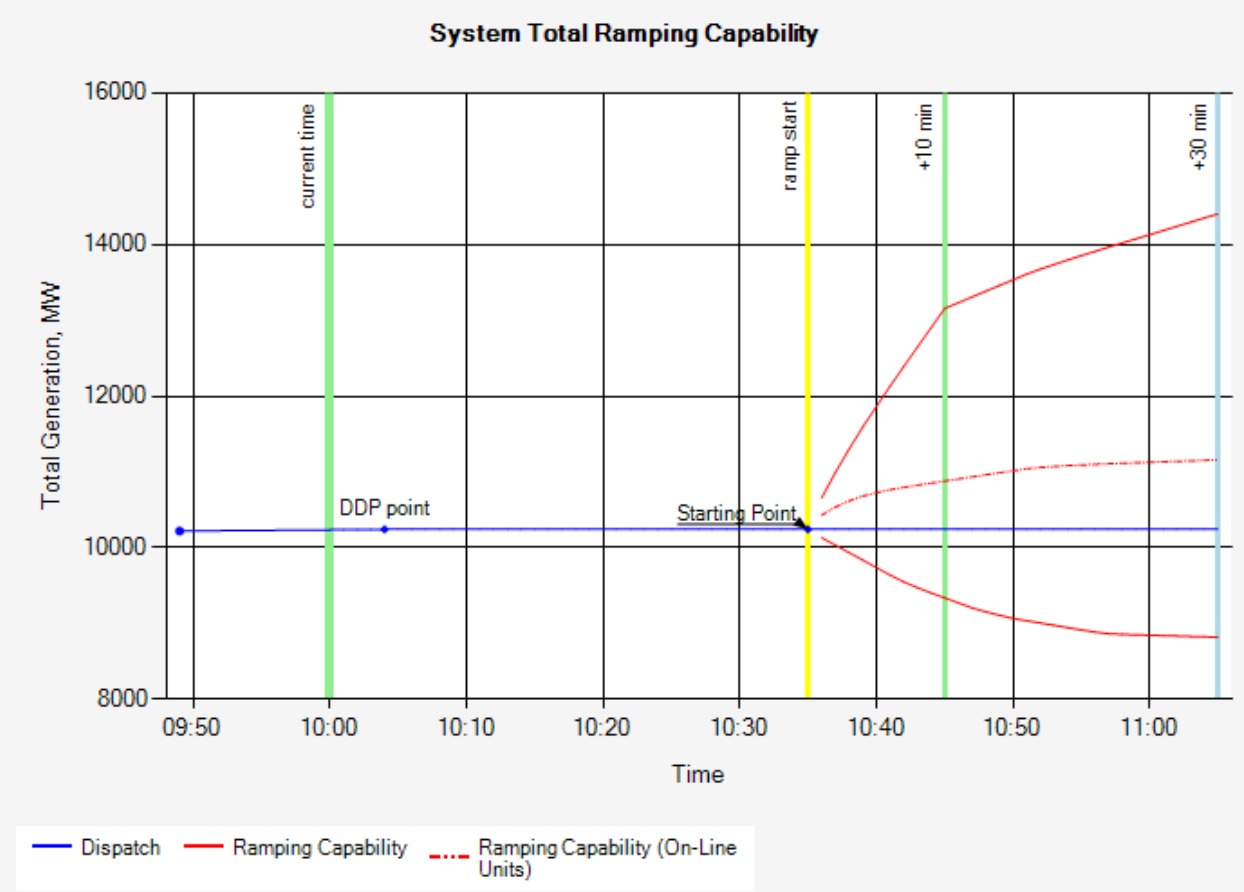

Figure 6-4. Total generation capability curves

\subsection{Secure Interchange Adjustment Range}

\subsubsection{System Ramping Capability}

Prediction of available ISO-NE system capability to securely adjust the interchange schedule is shown in Figure 6-5. Dashed black line shows predicted generation capability calculated at the previous step (see Section 6.1). Violet curve shows system capability taking into account reserve requirements and forecasted load variation. System capability for online and offline fast start unit is show in Figure 6-5 (a) and for online units only in Figure 6-5 (b). These curves are calculated deterministically and subject to certain inaccuracy due to lack of information on the generation schedule at the starting point and load forecast uncertainty. Actual (observed) system capability is calculated using the latest market available at starting point (10:35) and actual information on load and interchange variation, and depicted using red dots (Figure 6-5). The difference between red dots and violet curves is the prediction error caused by the aforementioned factors. 


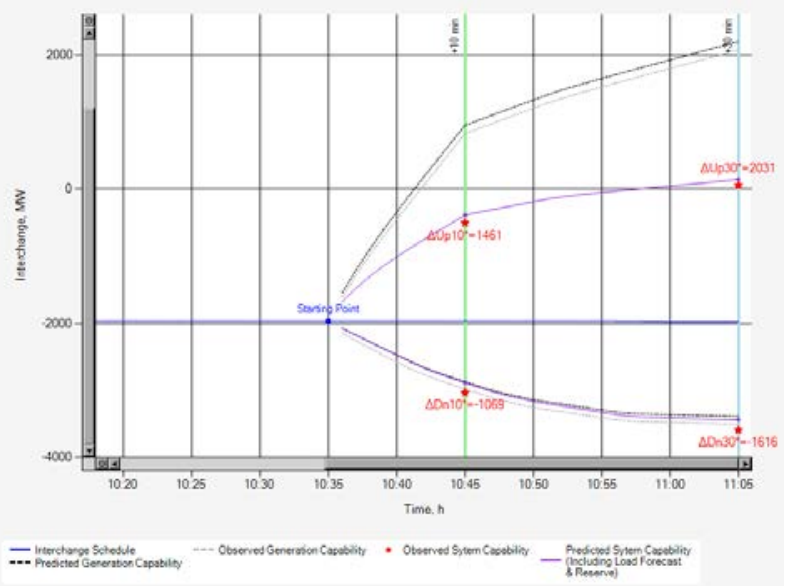

(a)

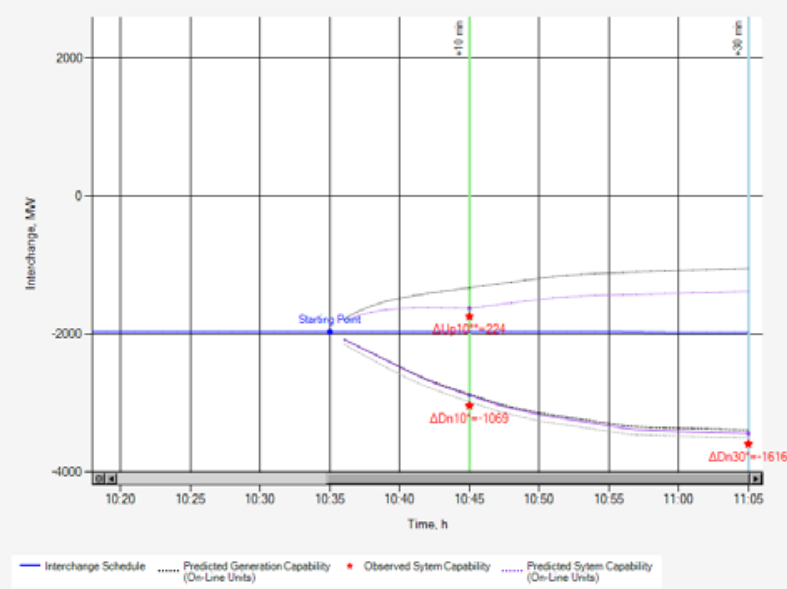

(b)

Figure 6-5. System capability: (a) Online and Offline units; (b) Online units only

\subsubsection{Prediction Errors Analysis}

The results of the analysis of the system capability prediction errors are presented in Table 6-3 and Figure 6-6. Performed analysis is hour-specific, where the prediction errors for user-specified time interval (e.g., 30 days) at operating interval ending by 11 are analyzed in this example. PDFs of the prediction errors for times $+10 \mathrm{~min}$ and $+30 \mathrm{~min}$ are shown in Figure 6-6. Percentiles corresponding to the 95\% confidence level are shown by red vertical lines.

Table 6-3. Prediction errors statistics

\begin{tabular}{|c|c|c|c|c|c|}
\hline & Up 10 & Up 10 (online only) & Down 10 & Up 30 & Down 30 \\
\hline Mean & 11 & 27 & -27 & 25 & 10 \\
\hline STD & 141 & 126 & 136 & 173 & 171 \\
\hline Min & -657 & -462 & -557 & -577 & -745 \\
\hline Max & 502 & 425 & 335 & 549 & 485 \\
\hline
\end{tabular}



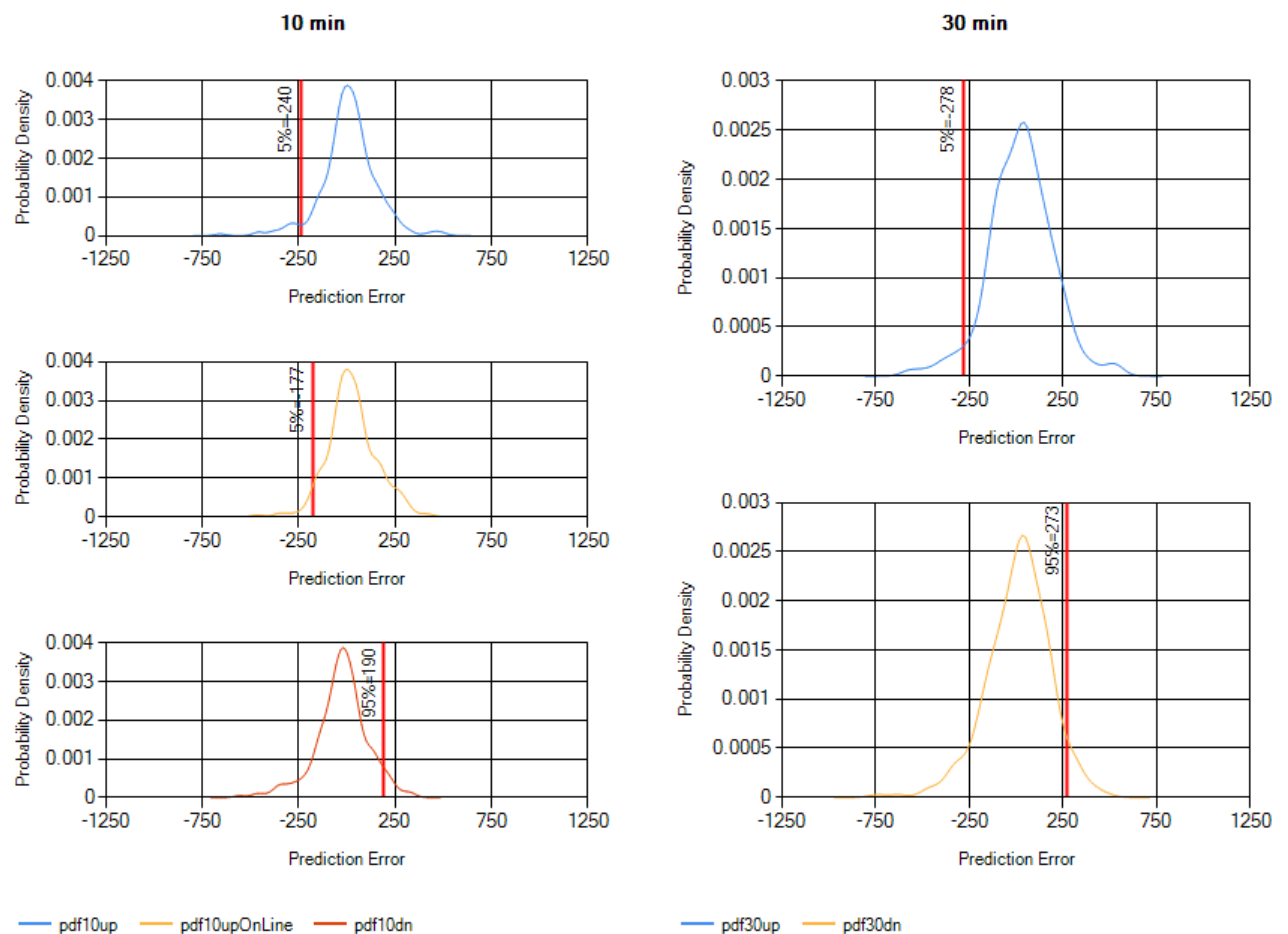

Figure 6-6. System capability prediction errors analysis

\subsubsection{Probabilistic System Capability}

At the next stage we take the plot from Figure 6-5 and add uncertainty ranges (with 95\% confidence) to the system ramping capability curves. These uncertainty ranges are shown in Figure 6-7 as orange areas. These ranges are based on the statistical analysis (PDFs) shown in Figure 6-6. The actual capability (red dots) is within predicted ranges with 95\% probability.

The secure range for interchange adjustment is shown using blue dots. For the case shown in Figure 6-7 at 10:35 a.m. interchange schedule can be adjusted $-730 \ldots+151 \mathrm{MW}$. If we also consider available offline fast-start units, the range can be extended to $-730 \ldots+1331$ MW. 


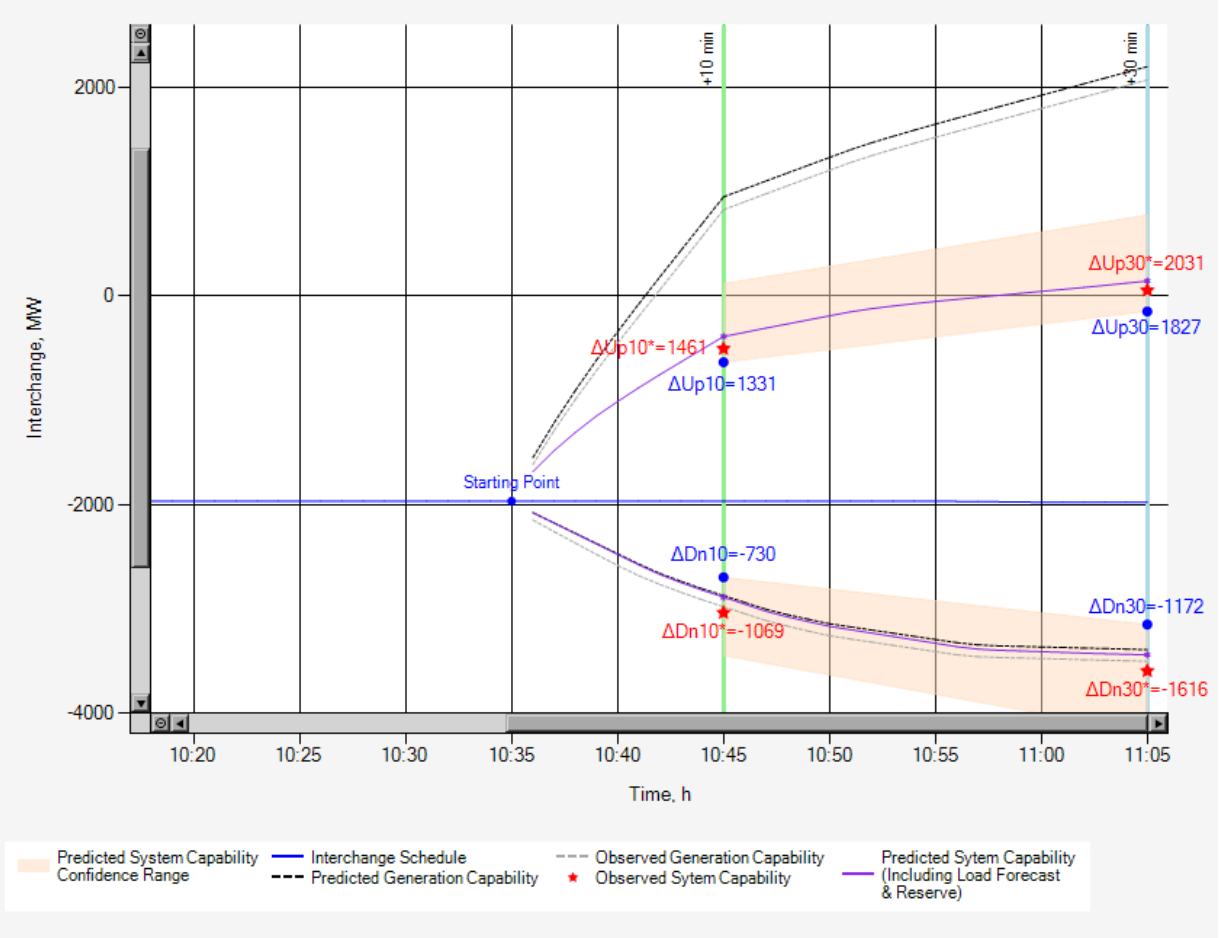

(a)

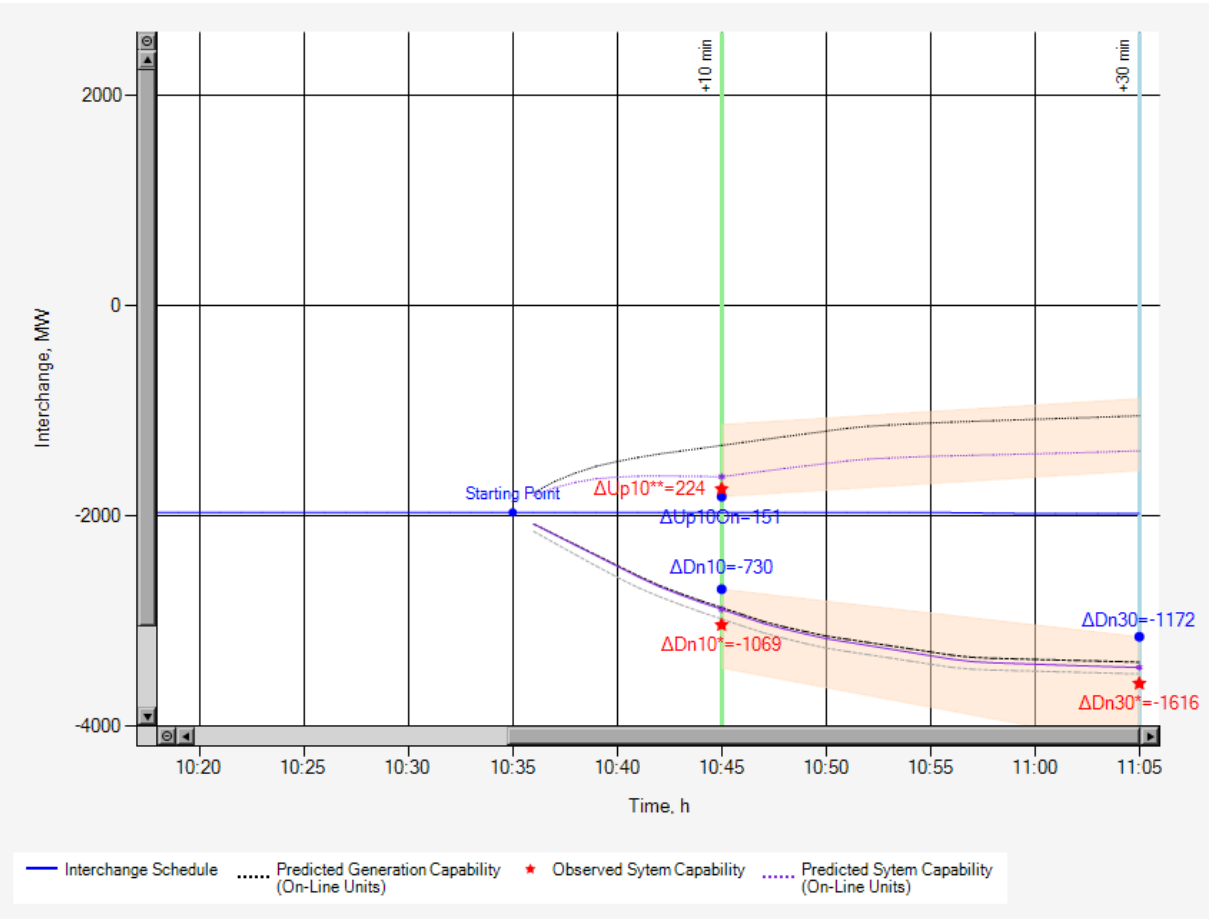

(b)

Figure 6-7. Secure Interchange adjustment range: (a) Online and Offline units; (b) Online units only 


\subsection{Self-Validation Results}

Results of self-validation for a 1-month interval are presented in Figure 6-8 . One can see that the observed percentage values are very close to the targeted percentage values. For example, the percentage of the actual ramping capability points found within the $95 \%$ confidence interval is $95.61 \%$ for +10 upward ranges, and the percentage within the $95 \%$ confidence interval is $94.52 \%$ for +30 min upward range.

Therefore, the uncertainty evaluation model tests have confirmed the consistency and reliability of the proposed uncertainty evaluation algorithm, as well as the proper functioning of the developed DINA tool.

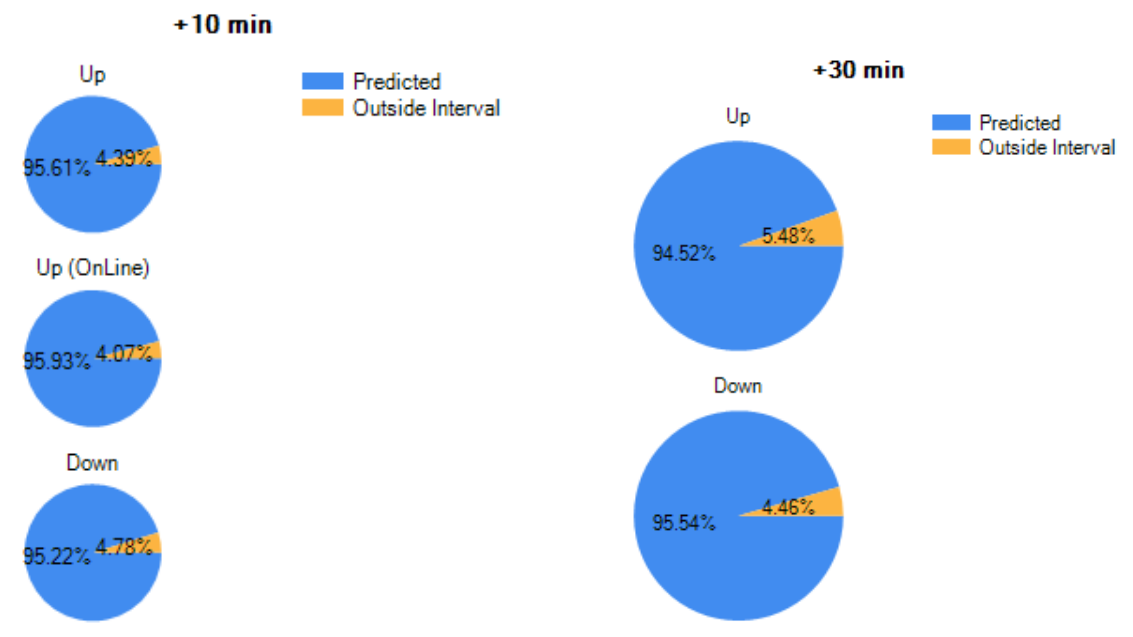

Figure 6-8. Self-validation results 



\subsection{Conclusions}

The project developed an uncertainty quantification methodology and practical tool for evaluating the secure range of ISO-NE dynamic intra-hour interchange adjustments in real time. The methodology considers the generation fleet ramping up and ramping down capability, as well as all uncertainties typical for the ISO-NE system. Results are provided to confirm the performance and accuracy of the DINA tool.

The initial version of the tool was installed at ISO-NE for testing in November 2012. Assuming the DINA tool is successful in the testing phase, ISO-NE can decide on integration of the tool into the ISONE's real-time market systems. 



\subsection{References}

[FERC 2012] “Integration of Variable Energy Resources”, Order No. 764, FERC, June 22, 2012. [Online.] Available: http://www.ferc.gov/whats-new/comm-meet/2012/062112/E-3.pdf

[ISO-NE Glossary] “Glossary \& Acronyms,” ISO-New England. [Online.] Available: http://www.isone.com/support/training/glossary/index-p1.html 



\section{Appendix A.}

\section{ISO-NE Ramping Capability Calculation Methodology}



The methodology for calculating ramping capability of the system reported in this Appendix was developed by ISO-NE.

\section{A.1 Calculation of Generating Units Ramping Capability (Not Including Pumping Units)}

1. $\mathrm{UCM}^{1}=6$ and 5 (exclude from 10 -minute ramping capability estimation)
a. Ramping up capability $=0$
b. Ramping down capability $=0$

2. $\mathrm{UCM}=4$

1) LEGFLAG $^{2}=0$
a. $10 \mathrm{~m}$ ramp-up capability $=\min \left\{\mathrm{CRR}^{3} * 10, \mathrm{AEMX}{ }^{4}-\mathrm{MW}\right\}$
b. $10 \mathrm{~m}$ ramp-down capability $=\min \left\{\mathrm{CRR}^{*} 10, \mathrm{MW}-\mathrm{AEMN}^{5}\right\}$
c. $30 \mathrm{~m}$ ramp-up capability $=\min \{\mathrm{CRR} * 30, \mathrm{AEMX}-\mathrm{MW}\}$
d. $30 \mathrm{~m}$ ramp-down capability $=\min \{C R R * 30, \mathrm{MW}-\mathrm{AEMN}\}$

2) LEGFLAG $=1$
a. $10 \mathrm{~m}$ ramp-up capability $=\min \{\mathrm{CRR} * 10, \mathrm{AEMX}-\mathrm{MW}, \mathrm{LEG}$ limit-MW $\}$
b. $10 \mathrm{~m}$ ramp-down capability $=\min \{\mathrm{CRR} * 10, \mathrm{MW}-\mathrm{AEMN}\}$
c. $30 \mathrm{~m}$ ramp-up capability $=\min \{\mathrm{CRR} * 30, \mathrm{AEMX}-\mathrm{MW}, \mathrm{LEG}$ limit-MW $\}$
d. $30 \mathrm{~m}$ ramp-down capability $=\min \{\mathrm{CRR} * 30, \mathrm{MW}-\mathrm{AEMN}\}$

3. $\mathrm{UCM}=3$
a. Ramping up capability $=0$
b. Ramping down capability $=0$

4. $\mathrm{UCM}=2$

1) Ramping down capability $=0$

2) $\mathrm{LEGFLAG}=0,10 \mathrm{~m}$ ramp-up capability $=\min \left\{\right.$ claim $10^{6}$, AEMX $\}$

3) $\mathrm{LEGFLAG}=0,30 \mathrm{~m}$ ramp-up capability $=\min \left\{\right.$ claim $30^{1}$, AEMX $\}$

\footnotetext{
${ }^{1}$ Unit control mode (UCM):

- $\quad \mathrm{UCM}=1$ - offline unit, not available

- $=2-$ offline/available

- $=3$ - online, non-dispatchable

- $=4$ online, dispatchable

- $=5$ - not used

- $\quad=6$ - regulating unit

${ }^{2}$ A flag for limited energy generators LEG (1 - LEG; 0 - Not LEG)

${ }^{3} \mathrm{CRR}$

${ }^{4}$ AEMX

${ }^{5}$ AEMN

${ }^{6}$ Claim 10 - The generation output level, expressed in megawatts, that a generator can reach from an off-line state within 10 minutes after receiving a dispatch instruction from ISO. This value is required as part of the generator's offer data and is used by ISO New England to evaluate the generator's contribution towards meeting operating- and replacement-reserve requirements [ISO-NE Glossary].
} 


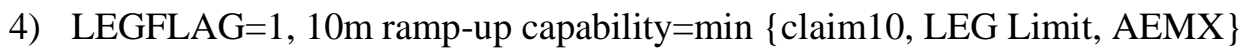

5) $\quad$ LEGFLAG=1, 30m ramp-up capability=min \{claim30, LEG Limit, AEMX $\}$

5. $\mathrm{UCM}=1$
a. Ramping up capability $=0$
b. Ramping down capability $=0$

\section{A.2 Special Case for Fast Start Generating Units}

1) When it is online (UCM changes from 2 to 4), once it meets minimum run time requirement, the ramping down capability is its MW output, instead of MW output minus AEMN as non fast start unit.

2) When it is offline $(\mathrm{UCM}=2)$, the ramping capability won't be counted until it meets the minimum down time requirements.

\section{A.3 Pumping Units}

1) Self-Schedule (see SELFSCHEDULE flag in the section 5.1 of data structure description)

a. Ramping up capability $=0$

b. Ramping down capability $=0$

2) Not in Self-Schedule

a. Ramping up capability = AEMX-MW

b. Ramping down capability $=-\mathrm{MW}-\mathrm{AEMN}$

\section{A.4 Requirement to Meet Reserves, Load Variation and Tie Line Ramping}

10m tie line ramping up capability = minimum (TMSR Surplus, T30 Surplus) - "10-min load forecast change at scheduled time”

1) TMSR Surplus = Generating units $10 \mathrm{~m}$ ramping up capability $(\mathrm{UCM}=4)+$ pumping unit ramping up capability - 10m spinning reserve requirement

2) T10 Surplus = Generating units $10 \mathrm{~m}$ ramping up capability $(\mathrm{UCM}=2$ and 4$)+$ pumping unit ramping up capability - Total $10 \mathrm{~m}$ reserve requirement

3) T30 Surplus = Generating units 30m ramping up capability $(\mathrm{UCM}=2$ and 4$)+$ pumping unit ramping up capability - Total 30 min reserve requirement

$10 \mathrm{~m}$ tie line ramping down capability = Generating units $10 \mathrm{~m}$ ramping down capability + pumping unit ramping down capability + "10-min load forecast change at scheduled time".

Note: 10 -min load forecast change at scheduled time $>0$ if load growths.

\footnotetext{
${ }^{1}$ Claim 30 - The generation output level, expressed in megawatts, that a generator can reach from an off-line state in 30 minutes after receiving a dispatch instruction from ISO. This value is required as part of the generator's offer data and is used by ISO New England to evaluate the generator's contribution towards meeting the New England Control Area's operating and replacement reserve requirements [ISO-NE Glossary].
} 


\section{Distribution}

No. of

Copies

\# Name

Organization

Address

City, State and ZIP Code

\# Organization

Address

City, State and ZIP Code

Name

Name

Name

Name

Name (\#)

\# Name

Organization

Address

City, State and ZIP Code
No. of

Copies

\# Foreign Distribution

\# Name

Organization

Address

Address line 2

COUNTRY

\# Local Distribution

Pacific Northwest National Laboratory

Name

Name

Name

Name

Name
Mailstop

Mailstop

Mailstop

Mailstop

(PDF) 




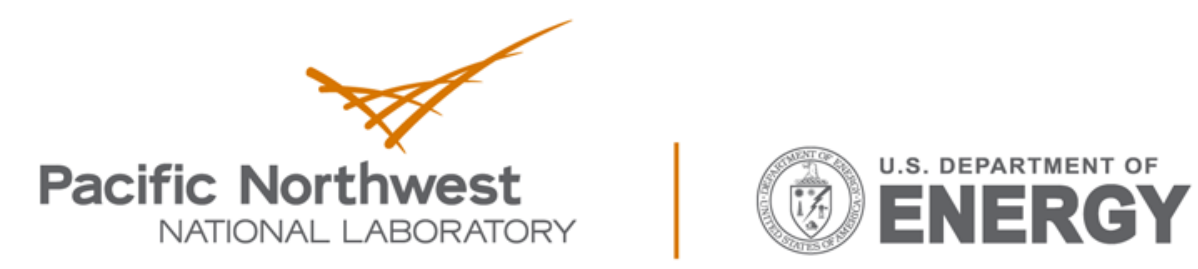

Proudly Operated by Battelle Since 1965

902 Battelle Boulevard

P.O. Box 999

Richland, WA 99352

1-888-375-PNNL (7665)

www.pnnl.gov 